\title{
Le problème du critère sceptique
}

\section{Baptiste Bondu}

\section{(2) OpenEdition}

\section{Journals}

Édition électronique

URL : https://journals.openedition.org/philosant/358

DOI : 10.4000/philosant.358

ISSN : 2648-2789

\section{Éditeur}

Éditions Vrin

\section{Édition imprimée}

Date de publication : 24 novembre 2015

Pagination : 53-90

ISBN : 978-2-7574-1141-4

ISSN : $1634-4561$

\section{Référence électronique}

Baptiste Bondu, « Le problème du critère sceptique », Philosophie antique [En ligne], 15 | 2015, mis en ligne le 01 novembre 2018, consulté le 02 décembre 2022. URL : http://journals.openedition.org/ philosant/358; DOI : https://doi.org/10.4000/philosant.358

Creative Commons - Attribution - Pas d'Utilisation Commerciale - Pas de Modification 4.0 International - CC BY-NC-ND 4.0

https://creativecommons.org/licenses/by-nc-nd/4.0/ 


\section{LE PROBLÈME DU CRITÈRE SCEPTIQUE}

Baptiste BONDU

Université Paris-Ouest-Nanterre-La Défense Paris 10

RÉSUMÉ. Comment concilier réfutation du critère par les sceptiques et adoption d'un critère par les sceptiques ? La première partie de l'article revient sur les formulations de la réfutation du critère dans les différentes sources sur le scepticisme et expose la manière dont Sextus Empiricus présente le scepticisme moins comme une négation de l'existence du critère que comme une mise en suspens sur la nature et l'existence du critère dogmatique. La deuxième partie de l'article rappelle les passages, de Cicéron à Galien, en passant par le Commentateur anonyme du Théétète et Diogène Laërce, qui attribuent plus ou moins explicitement quelque chose comme un critère aux sceptiques. Plus précisément, ce critère, comme l'énoncera de la manière la plus claire Sextus Empiricus, est un critère d'action distinct d'un critère de vérité, cette distinction valant comme un moyen de faire la part entre scepticisme et dogmatisme mais aussi comme un argument décisif pour répondre aux objections dogmatiques contre le scepticisme. La troisième partie de l'article est consacrée à un approfondissement de la compréhension du critère sceptique comme critère pratique. Les textes de Sextus Empiricus permettent de montrer en quoi cette conception ne doit pas être confondue avec la restriction du critère au strict domaine de la conduite de la vie, ni avec une manière d'user de la vie ordinaire comme critère de vérité.

SUMMARY. How can the sceptical refutation of the criterion be reconciled with the sceptical adoption of a criterion? The first part of the paper starts from the different expressions of the sceptical refutation of the criterion in ancient sources and exhibits how Sextus Empiricus presents scepticism not as much as a negation of the criterion's existence as a suspension about the nature and existence of a dogmatic criterion. The second part of the paper recalls the texts which, from Cicero to Galen including the Anonymous commentator on the Theaetetus and Diogenes Laertius, attribute more or less explicitly something like a criterion to the sceptics. More precisely, this criterion, as Sextus Empiricus formulates it in the clearest manner, is a criterion of action that is distinct from a criterion of truth, this distinction being altogether a means to make the difference between scepticism and dogmatism and a decisive reply to the objections of the dogmatists against scepticism. The third part of the paper is dedicated to a deeper understanding of the sceptical criterion's identification to a practical criterion. Sextus Empiricus'texts allow us to show that such a conception is not to be confused with the

Philosophie antique, $\mathrm{n}^{\circ} 15$ (2015), 53-90 
view that would restrict the use of a criterion only to matters concerning the way one should conduct one's life, nor with the consideration that ordinary life is the criterion of truth. 
La notion de critère, translittération du grec kpıtท่pıov, désigne techniquement dans la philosophie ancienne, et tout particulièrement dans la philosophie hellénistique, la base irréfutable sur laquelle se fonder pour établir une connaissance ${ }^{1}$. En ce sens, elle constitue le cœur de la réflexion épistémologique après Platon et Aristote: comme on l'exprime parfois, avec la question du critère, on passerait de la question « qu'est-ce que le savoir, par différence avec l'ignorance ? » à la question « est-ce que le savoir existe ? $\gg^{2}$. Ainsi toute école philosophique de la période hellénistique se doit de dire quel est le critère sur lequel elle fonde sa doctrine. On a pu ainsi considérer que cet infléchissement du questionnement dans la philosophie ancienne de la connaissance est lié à la prise au sérieux de l'objection « sceptique » selon laquelle savoir et ignorance se confondent, ce qui rendrait impossible de choisir l'un à la place de l'autre - le scepticisme pouvant alors être défini de manière large comme la remise en cause de la capacité de savoir ${ }^{3}$. Épicuriens et stoïciens auraient alors proposé de partir préalable-

1. Sur cette notion, voir l'article de référence, Striker 1974. Long 1978 (p. 35) propose la définition générale suivante: «a "criterion of truth" is a means of judging correctly that something is the case ». S'appuyant sur les occurrences du terme chez Sextus Empiricus, Annas 1990 (p. 189 n. 18) décrit un champ sémantique assez large, qui va de ce que l'on use ou suit, à ce qui permet de dire telle ou telle chose, de juger, de distinguer, d'examiner, ou ce à quoi on peut se fier : le sens général demeure bien ce qui permet de dire ce qui est vrai ou faux, soit la base de la constitution d'une connaissance. $C f$. Janáček 1972, p. 67-72, qui met en parallèle des textes de Sextus Empiricus permettant de rapprocher la notion de critère de



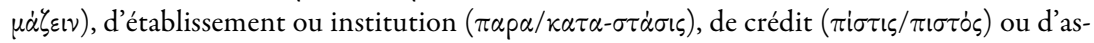
surance ( $\beta \dot{\varepsilon} \beta \alpha 10 \varsigma)$.

2. Pour une analyse de la valeur de la notion dans la période antérieure et postérieure de la philosophie ancienne, voir toutefois Huby et Neal (éd.) 1989. La période post-hellénistique ou impériale (celle de Sextus Empiricus, Diogène Laërce, Galien, Ptolémée et Alexandre d'Aphrodise) fut particulièrement préoccupée par la question du critère : voir Long 1989.

3. Striker 1990, p. 143-144, écrit ainsi : « There must have been a sceptical undercurrent from the time of the sophists on, most notably perhaps in the Democritean school. (...) Plato and 
ment d'une clarification de la nature des données et des sources pouvant être considérées comme fiables afin de pouvoir édifier, dans un second temps, le système global de connaissance qu'est le savoir. Dans cette perspective, on pourrait dire que c'est un questionnement de type sceptique qui est à l'origine de la notion de critère telle qu'elle s'est imposée dans le débat philosophique comme objet liminaire de la réflexion et condition de possibilité de l'ensemble de l'édifice du savoir. Mais, tandis que la réponse philosophique à cette première objection de type sceptique s'est incarnée dans la poursuite du critère ou fondement de la connaissance, est apparue une nouvelle argumentation, destinée en retour à remettre en cause toute forme de critère, et constituant une nouvelle base pour le scepticisme. La réfutation du critère constitua ainsi comme un passage obligé du scepticisme hellénistique ${ }^{4}$. Tout un arsenal d'arguments a été déployé soit pour rendre la notion elle-même invalide soit pour contester telle ou telle forme particulière de critère, et en particulier la raison ( $\lambda$ óyos) des


appréhensive ( $\phi \alpha \nu \tau \alpha \sigma i \alpha \kappa \alpha \tau \alpha \lambda \eta \pi \tau i \kappa \eta \dot{)}$ ) des disciples de Chrysippe. Ce qui pose davantage difficulté, et sur quoi les sources sont moins concordantes, est de savoir si les sceptiques, tout en annihilant toutes les formes « dogmatiques » de critère, n'ont pas néanmoins formulé en propre une espèce de critère qu'ils auraient acceptée 5 . On pourra penser que les sceptiques ont été contraints de formuler l'adoption d'un critère sous le coup de la double objection classique de l'impossibilité logique et de l'impossibilité vitale. L'impossibilité logique de la réfutation du critère peut s'exprimer ainsi : si le sceptique réfute tout critère, sur quelle base, et donc sur quel critère, pourra-t-il effectuer cette réfutation ? L'impossibilité vitale, quant à elle, peut prendre la forme suivante : si le sceptique réfute tout critère, sur quelle base, et donc sur quel critère, pourra-t-il mener sa vie ? La formulation d'un critère sceptique serait donc un moyen de sauver le scepticisme de ses inconséquences. La question est alors de savoir si, en l'occurrence, le remède n'est pas pire que le mal. En effet, à partir du moment où un sceptique

Aristotle (...) had little patience with doubts about the possibility of knowledge. (...) But the fourth century also produced Pyrrho, later seen as the founder of scepticism, by whom Epicurus (...) is said to have been much impressed (DL IX 64). Hence it is not surprising to find the major Hellenistic philosophers preoccupied with the task of justifying their claims to knowledge. »

4. Voir Striker 1990, qui étudie successivement la version académiciennne et la version pyrrhonienne de cette réfutation sceptique du critère.

5. L'idée de critère sceptique apparait explicitement chez Diogène Laërce, IX 105, dans le Commentaire anonyme sur le Théétète, 61.15-22 et plusieurs fois chez Sextus Empiricus : Esquisses Pyrrhoniennes [= PH] I 22 et II 15; Contre les logiciens [= M VII] 29-30, 158 et 166. 
propose une forme de critère qu'il peut accepter, ne retombe-t-il pas d'emblée dans le dogmatisme qu'il reproche précisément aux autres philosophes ? Le scepticisme ne réside-t-il pas dans la radicalité de sa proposition philosophique, aboutissement d'une argumentation implacable contre toute prétention à établir des fondements certains pour la connaissance et, partant, pour la vie elle-même ? Le fait de s'en remettre à un critère, fût-il considéré de la manière la plus précautionneuse et la plus provisoire possible, ne conduit-il pas à un amoindrissement de la portée du scepticisme tout en n'atteignant pas la vigueur et la cohérence des systèmes philosophiques qu'il combat ${ }^{6}$ ?

De manière liminaire, nous parlerons de sceptiques et de scepticisme en un sens générique pour désigner, selon l'usage le plus ordinaire chez les interprètes contemporains, le courant de pensée qui va de Pyrrhon à Énésidème et Sextus Empiricus, en passant par Arcésilas et Carnéade, pour ne citer que les figures les plus importantes, et sans préjuger des débats, anciens et modernes, sur les différentes versions de ces scepticismes et même sur la légitimité de cette appellation pour chacun de ces philosophes ${ }^{7}$. Mais il s'agit d'un problème d'histoire de la philosophie autant que de philosophie. En effet, d'une part, une analyse des textes et des contextes dans lesquels est énoncée l'existence d'un critère sceptique doit nous permettre d'évaluer la part de la dimension polémique et de la déformation doxographique dans une telle assertion: en d'autres termes, est-ce que l'affirmation selon laquelle le sceptique a lui aussi un critère peut être considérée comme authentiquement sceptique ou n'est-elle qu'une façon ad hoc de dire les choses pour répondre à une objection ou pour donner à cette position philosophique une coloration qui la rapproche des autres doctrines ? Une question liée est celle de savoir si toutes les formes de scepticisme de la période hellénistique, des académiciens aux néopyrrhoniens, ont défendu cette position et si elles l'ont fait de la même manière. Mais, d'autre part, prendre au sérieux la thèse selon laquelle un sceptique peut se fonder sur un critère impose de définir précisément la nature du critère en question et, symétriquement, la nature du scepticisme en question, quitte à considérer que certaines formes de pensée ne sont précisément plus «sceptiques». Plus précisément, est-ce que le critère du sceptique est du même type que le

6. Voir Barnes 1982, 1990, 2007, qui distingue entre scepticisme policé (urbane) et scepticisme enragé ( $\mathrm{rabid}$ ), l'un et l'autre conduisant à d'inextricables impasses.

7. Le plus fameux de ces débats porte sur le point de savoir si les académiciens Arcésilas et Carnéade sont bien des sceptiques comme les pyrrhoniens qui, comme Énésidème et Sextus Empiricus, se réferent à Pyrrhon; mais la question est aussi de savoir si Pyrrhon luimême, voire Énésidème, sont des sceptiques, Sextus Empiricus demeurant alors le seul auteur authentiquement sceptique. Voir, déjà, Brochard 1887, mais surtout Hankinson 1995, Lévy 2008, Bett 2010. 
critère d'un philosophe dogmatique, épicurien ou stoïcien notamment, mais en diffère par son contenu, de la même manière que le critère épicurien qu'est la sensation se distingue du critère stoïcien qu'est l'impression appréhensive ? Ou bien est-ce que le critère du sceptique n'est tout simplement pas du même type que le critère d'un philosophe dogmatique, et que ce n'est finalement que par équivoque ou homonymie que l'on parle alors de « critère » dans les deux cas, le mot ne désignant pas la même chose chez le sceptique et chez le dogmatique? Dans cette dernière hypothèse, comment faudra-t-il comprendre la notion de critère pour un sceptique, par différence avec la notion de critère pour un dogmatique ? On pourra ainsi parler de manière générale d'un « problème du critère sceptique $\gg$, d'une part, au sens où l'identification de la nature de ce critère est complexe mais aussi, d'autre part, au sens où la possibilité même d'un tel critère est discutable. Une grande difficulté pour l'analyse de ce problème vient du fait que, dans les sources anciennes elles-mêmes, les formulations attribuées aux sceptiques ne sont pas toujours cohérentes avec ce que l'on attendrait du scepticisme ou pas toujours concordantes entre elles. Davantage, les sources tendent à confondre dans une grande catégorie générale « les sceptiques », sans distinguer entre les différents penseurs ou les différentes traditions, et, quand ces distinctions sont apparemment opérées, sans que les sources puissent être considérées comme parfaitement fiables et sans parti pris. L'objectif des lignes qui vont suivre est de tenter d'y voir plus clair dans la série de problèmes suscités par l'hypothèse d'un critère sceptique et dans la manière dont la question a été abordée par certains auteurs de l'Antiquité, et en particulier celui dont les réflexions sur le sujet restent pour nous les plus fournies, à savoir Sextus Empiricus ${ }^{8}$.

\section{La réfutation sceptique du critère}

Commençons par recenser et examiner les différentes manières dont les sources antiques ont présenté le rapport des sceptiques à la question du critère. Il conviendra à cet égard de distinguer selon les sources mais aussi selon les sceptiques qui sont alors étudiés. En effet, les textes nous présentent une assez grande variété de formulations, lesquelles renvoient à proprement parler à des options philosophiques distinctes qui ne sont pas

8. On passera alors d'une acception très large du scepticisme à une version plus étroite, celle de Sextus Empiricus et même de Sextus Empiricus dans certains de ses textes: je remercie le relecteur anonyme de cet article d'avoir souligné cette difficulté, qui permet de s'interroger sur l'unité même du « scepticisme » comme courant philosophique unifié. Sur cette difficulté à concevoir le scepticisme comme courant historique continu, lequel serait seulement une reconstruction tardive, voir en particulier Pellegrin 2010, p. 122. 
toujours clairement identifiées et qui restent généralement confusément désignées sous le nom de «scepticisme». Revenir sur la conception sceptique du critère permet ainsi de revenir sur les différentes formes de scepticisme qui s'exprimèrent dans la philosophie ancienne, même si ces distinctions s'avèrent elles-mêmes discutées, tant par les auteurs anciens que par les interprètes contemporains. Tous les passages qui évoquent la manière sceptique de considérer le critère sont unanimes sur un point : la notion philosophique de critère a été l'objet d'une réfutation générale par les sceptiques. Les passages les plus explicites sont ceux que l'on trouve chez Sextus Empiricus et Diogène Laërce. Le premier déploie la version la plus étendue de l'argumentation sceptique contre le critère, y consacrant une grande partie du deuxième livre des Esquisses pyrrhoniennes (PH II 14-79) et pas moins de quatre cents paragraphes du Contre les logiciens ( $M$ VII 46446), la première partie (46-260) étant vouée à présenter le désaccord

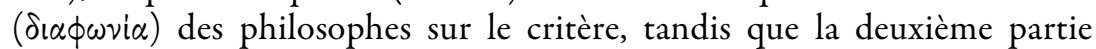
(261-446) expose une succession d'arguments contre le critère9. Le second (DL IX 90) commence par annoncer que le critère fait partie des notions que les sceptiques «éliminèrent » (¿่vñpovv), au même titre que la démonstration, le signe, la cause, le mouvement ou le bien et le mal. Puis (DL IX 94) il offre un rapide exemple de l'argumentation systématique qui était alors déployée par les sceptiques contre le critère, sous la forme de l'examen d'alternatives successivement déboutées ${ }^{10}$ - avant (DL IX 95) de compléter cette argumentation par une autre fondée sur le désaccord $(\delta 1 \alpha-$ $\phi \omega v i \alpha)^{11}$. Diogène Laërce parle ici du scepticisme sous l'égide de la figure de

9. Voir Long 1978; Hankinson 1995, p. 193-200.

10. DL IX $94:$ : Ou bien le critère est adopté par un critère ou bien il est adopté sans

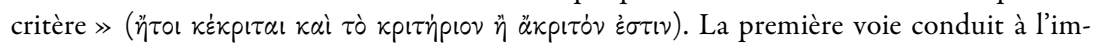
passe de la réciprocité et de la régression à l'infini, la seconde renvoie à la pétition de principe ou postulat infondé. Sextus rapporte la tentative dogmatique d'échapper à cette impasse, en posant que le critère, parce qu'il est la base première, n'a pas lui-même besoin de critère ( $M$ VII $441-442 ; c f$. Épictète, Entretiens, II $11, \$ 14-15)$ : il y répond de même par le renvoi à une régression à l'infini ou à une pétition de principe sans fondement argumentatif valable.

11. Ce dernier argument, qui se fonde sur le désaccord à propos du critère entre l'hom-

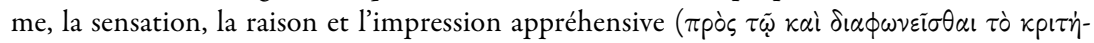

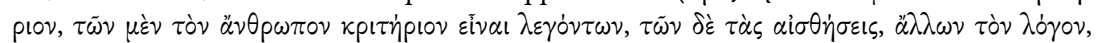


tion que l'on trouve dans le Contre les logiciens, la première partie ( $M$ VII 46-260) visant à montrer le désaccord entre ceux qui nient le critère, ceux qui le placent dans la raison, ceux qui le placent dans la sensation et ceux qui le placent dans l'impression, et la deuxième partie ( $M$ VII 261-439) qui, parallèlement à $P H$ II 22-78, propose une analyse successive des arguments contre le critère conçu comme sujet ( $\left.\dot{\phi} \phi^{\prime} \circ{ }^{\prime}\right)$, soit principalement l'homme ( $M$ VII 263-342; PH II 22-47), contre le critère conçu comme moyen ( $\left.\delta l^{\prime} \circ \tilde{u}\right)$, soit la 
Pyrrhon, et Sextus Empiricus se présente lui-même comme un représentant du pyrrhonisme.

Mais la réfutation du critère est partagée avec les sceptiques académiques. Ainsi Sextus écrit-il à propos d'Arcésilas qu'il « ne définissait

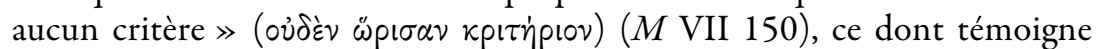
l'argumentation rapportée alors par Sextus ( $M$ VII 150-158). Sextus est tout aussi clair à propos de Carnéade, dont il expose de manière développée les arguments contre le critère ( $M$ VII 159-165) et dont il dit qu'il con-

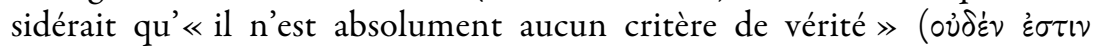
$\dot{\alpha} \pi \lambda \tilde{\omega} \varsigma \dot{\alpha} \lambda \eta \theta \varepsilon i \alpha \varsigma$ кpı $\tau \dot{\eta} p ı \nu)$ ( $M$ VII 159) ou qu'il « argumenta sur l'inexis-

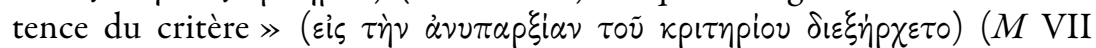
166). Quand il aborde la question dans son petit traité Sur le meilleur enseignement (Kühn I 40-52), Galien associe d'ailleurs «académiciens et pyrrhoniens » (I 42, I 48) dans cette réfutation de tout critère. Et si les débats des Académiques de Cicéron, tout particulièrement le Lucullus, tournent plutôt autour de la notion d'appréhension (comprehensio, perceptio et cognitio traduisant le grec $\kappa \alpha \tau \dot{\alpha} \lambda \eta \psi\left(\varsigma^{12}\right)$, il y est bien question du problème du critère et de la possibilité ou non de tenir une position sans critère de vérité, comme l'indique ponctuellement l'usage du terme judicium, équivalent latin de $x p \iota \eta_{p} \iota^{13}{ }^{13}$. Renvoyant lui aussi à la notion d'appréhension, Photius introduit sa présentation des Discours pyrrhoniens d'Énésidème en des termes qui peuvent être relus comme l'expression d'une réfutation de tout critère : « Le projet général du livre est d'assurer qu'il n'y a rien de sûr

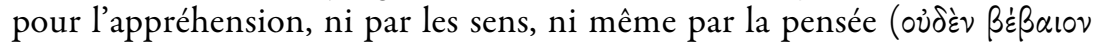

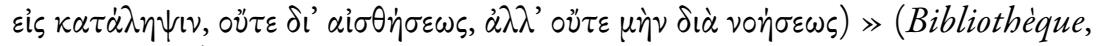
$212,169 \mathrm{~b} 20)$. Et, pour finir ce tour d'horizon des sources présentant la position du scepticisme sur le critère, on pourra également citer ce passage d'Eusèbe de Césarée : « et il y eut Pyrrhon, à partir duquel se constitua le groupe de ceux qui ont été nommés “sceptiques" - lesquels considéraient qu'absolument rien n'est susceptible d'appréhension, ni dans la sensation

sensation et la raison ( $M$ VII 343-369; $P H$ II 48-69), et contre le critère conçu comme référence ( $\kappa a \theta^{\prime}$ 'ö), soit notamment l'impression appréhensive ( $M$ VII $370-439$; $P H$ II 70 78).

12. Voir Ac. Pr. II, 17 et II, 31, où Cicéron dit que comprehensio est la traduction verbum e verbo de katalepsis. En De Fin. III, 17, il écrit : rerum autem cognitiones quas vel comprehensiones vel perceptiones vel, si haec verba aut minus placent aut minus intelleguntur, catalepsis appellemus licet. Je remercie Jean-Baptiste Gourinat pour ces références exactes.

13. Cicéron, $A c$. II 18 : « quand Philon affaiblit et se débarrasse de cela, il se débarrasse aussi du critère du connu et de l'inconnu. Le résultat est que rien n'est appréhendable - et il retombe alors sans le vouloir dans la position qu'il essayait d'éviter. » Voir Brittain 2006, p. 12-13 et surtout XIX-XXXV. 


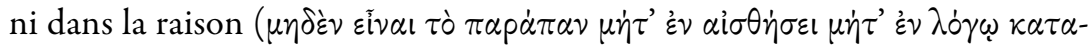

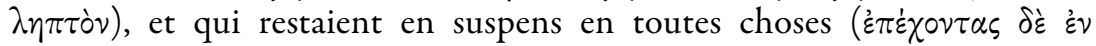
$\pi \tilde{\alpha} \sigma \nu) \gg$ (Préparation évangélique, XIV 17, \$10). Ce passage introduit les chapitres qu'Aristoclès de Messine a consacré aux différentes thèses sur le critère de vérité, chapitres qui contiennent notamment le fameux passage dans lequel Timon de Phlionte, disciple de Pyrrhon d'Élis, aurait exposé la thèse de son maître, thèse qui renvoie d'ailleurs elle-même, selon certains commentateurs, à une position sur le critère de véritét ${ }^{14}$.

Avant d'en venir à la difficulté principale qu'est la conciliation de cette réfutation du critère, dont toutes les sources s'accordent à faire un attribut essentiel du scepticisme, d'une part, et de l'acceptation d'un critère par les sceptiques, d'autre part, il convient de s'arrêter sur une difficulté préalable. Par leur réfutation du critère, les sceptiques en viennent-ils à nier le critère, c'est-à-dire à en contester absolument la réalité ? À première vue, la réponse à cette question va de soi : tout cet ensemble d'arguments contre le critère serait-il déployé en vain ? Le but du scepticisme n’est-il pas précisément de détruire toute prétention à se fonder sur un critère ? Il semble pourtant nécessaire d'être attentif au détail des formulations sur ce point. Si l'on repart des sources que nous venons de citer, on trouvera les expressions suivantes : les sceptiques tantôt « éliminent » le critère (DL IX 90), « argumentent en faveur de l'inexistence du critère » (SE $M$ VII 166), ou «se débarrassent du critère » (Cic. $A c$. II 18), tantôt « assurent qu'il n'y a rien de sûr pour l'appréhension » (Photius, Bibliothèque, 212, 169b20) ou « considèrent qu'absolument rien n'est susceptible d'appréhension » (Eusèbe, Prép. év. , XIV 17, \$10), tantôt « ne définissent aucun critère » (SE $M$ VII 159) ou « restent en suspens en toutes choses » (Eusèbe, Prép. év. , $\mathrm{XIV}, 17, \S 10)$. Or ces formulations n'ont pas du tout la même portée : elles tendent parfois vers une remise en cause radicale du critère, parfois vers une forme de certitude sur le caractère incertain du critère, parfois vers une simple confession d'ignorance sur l'existence ou non du critère. Sur cette base, on pourra donc distinguer entre trois formes de scepticisme, soit un

14. Un débat divise depuis longtemps les interprètes sur le texte en XIV 18, $\$ 2$-4. La question essentielle est de savoir s'il convient de comprendre, à partir de ce passage, que la position de Pyrrhon était avant tout métaphysique (sur l'indétermination des choses) ou si elle était avant tout épistémologique (sur l'indétermination du savoir) : dans le premier cas, la question du critère n'est pas vraiment abordée par Pyrrhon ou seulement comme conséquence; dans le deuxième cas, la question du critère est au cœur. Une question annexe est de savoir si ce qui est rapporté alors est fidèle à Pyrrhon ou s'il s'agit d'une retranscription en termes déjà hellénistiques. Pour une présentation de ce débat, voir Brennan 1998 (partisan de la lecture épistémologique, et suggérant qu'il s'agit d'une reformulation postérieure) contra Bett 2000, notamment chapitre I, p. 14-62 (partisan de la lecture métaphysique, et posant que le texte est fidèle à la pensée de Pyrrhon). 
scepticisme nihiliste (il n'y a pas de critère), un scepticisme indéterministe (le critère est quelque chose d'incertain) et un scepticisme suspensif (je ne sais s'il y a un critère ou non et s'il est incertain ou non).

Cette confusion sur la détermination précise de l'attitude des sceptiques à l'égard du critère, dont témoigne parfois un même auteur à quelques lignes d'intervalle, est à l'origine de controverses importantes sur la nature même du scepticisme, et ce dès l'Antiquité. Pour une version contemporaine de ces débats, on pourra renvoyer aux positions contradictoires défendues par Karel Janáček, d'une part, et Anthony Long, d'autre part, à propos du même auteur sceptique, à savoir Sextus Empiricus. Pour le premier, il n'y a pas de doute sur le fait que le scepticisme conduit à une pure et simple destruction du critère : c'est ce que prouveraient l'emploi de formes uniquement négatives pour mettre en évidence trois conclusions successives à propos du critère : son caractère inappréhendable, son caractère inexistant et son caractère inadoptable ${ }^{15}$. Pour le second, une telle interprétation est erronée et méconnaît les textes mêmes de Sextus Empiricus, lequel se prémunit explicitement contre l'assimilation du scepticisme à un nihilisme, notamment dans les paragraphes qui viennent conclure la réfutation du critère tant dans les Esquisses pyrrhoniennes que dans le Contre les logiciens $^{16}$. Le problème du rapprochement ou non des sceptiques et de ceux qui nient le critère se loge jusque dans les manuscrits des textes de Sextus Empiricus, le texte du Contre les logiciens transmis par la tradition rangeant en $M$ VII 48 les sceptiques ( $\kappa \alpha i$ oi $\dot{\alpha} \pi \grave{o} \tau \tilde{\eta} \varsigma \sigma \kappa \varepsilon \dot{\psi} \varepsilon \omega \varsigma$ ) parmi les penseurs qui

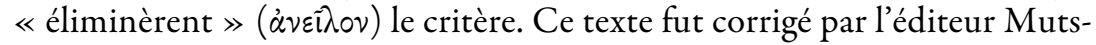
chmann depuis le début du $\mathrm{XX}^{\mathrm{e}}$ siècle - et conservé depuis par les traducteurs successifs -, notamment sur la foi du passage parallèle des Esquisses (PH II 18), qui distingue clairement entre ceux qui disent que le critère existe, ceux qui affirment qu'il n'existe pas, et ceux qui restent en suspens sur la question de savoir s'il existe ou non. Mais cette hésitation

15. Voir Janáček 1972 : p. 63 («from the point of view of the sceptics only the negative forms are used: there is no criterion, we cannot judge, we cannot prefer something to something,

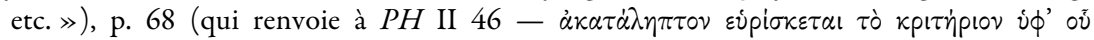

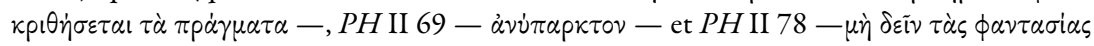

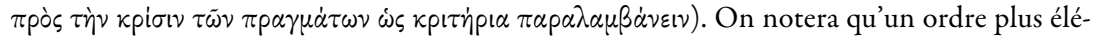
gant de l'argumentation serait, sur le modèle de l'argument de Gorgias transmis par Sextus ( $M$ VII 65-87) de montrer tout d'abord l'inexistence du critère, puis, après concession de son existence, son caractère inappréhendable, et enfin, après concession de son caractère appréhendable, son caractère inadoptable.

16. Long 1978, p. 36 : «In concluding his refutation of dogmatic claims about the criterion of truth Sextus states explicitly that he has not intended to show that no such thing exists (PH 2.79, M 7.443). 》 
entre les textes ne traduit-elle pas une ambiguïté profonde de la position des sceptiques face au critère, et de Sextus Empiricus en particulier ?

Pour répondre à cette question, on commencera par revenir sur les formulations que l'on trouve chez Sextus Empiricus, ses ouvrages proposant l'argumentation sceptique la plus développée et la plus précise qu'il nous reste à propos du critère. Or un tel examen détaillé des deux passages du corpus de Sextus Empiricus consacrés au critère ( $P H$ II 17-79 et $M$ VII 261-439) conduit à plusieurs remarques. Tout d'abord, on peut noter une certaine différence de tonalité entre le passage des Esquisses et celui du Contre les logiciens : le premier conduit plus facilement à identifier le scepticisme à une négation du critère que le second, ce qui permet d'expliquer un peu la divergence de lecture entre Janáček et Long, le premier auteur ne s'appuyant alors que sur $P H$, tandis que le second interprète consacre précisément son article à l'ouvrage moins connu qu'est $M$ VII. Deux aspects des passages en question vont dans ce sens. Premièrement, alors que dans

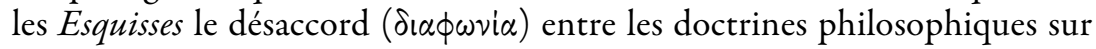
l'existence et la nature du critère est conçu d'emblée comme un argument contre le critère et fait pleinement partie de la réfutation $(\dot{\alpha} \nu \tau i p p \eta \sigma ı)^{17}$, dans le Contre les logiciens le désaccord ( $\delta \iota \alpha \omega \nu i \alpha)$ entre les doctrines constitue plutôt la partie positive qui précéde la réfutation ( $\dot{\alpha} \tau i p p \eta \sigma \varsigma)$ proprement dite $^{18}$ : ainsi la présentation des Esquisses ne laisse aucune place à la défense du critère, contrairement à celle du Contre les logiciens. Deuxièmement, dans les Esquisses, Sextus introduit sa réfutation du critère en écrivant que ses arguments permettront d'établir l'inappréhension ( $\alpha \kappa \alpha \tau \alpha-$ $\lambda \eta \psi i \alpha)$ du critère (PH II 21); il conclut la première partie de sa réfutation en disant qu'il en ressort que «le critère se trouve inappréhendé ( $\dot{\alpha} \kappa \alpha \tau \dot{\alpha}-$ $\lambda \eta \pi \tau \circ v) \gg(P H$ II 46); et il conclut la deuxième partie de sa réfutation en

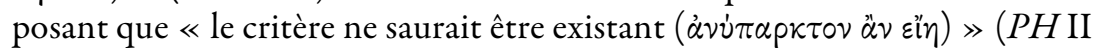

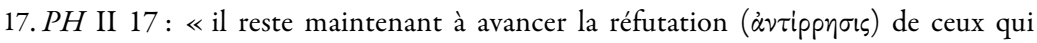
disent de manière précipitée avoir appréhendé le critère de vérité, et ce en commençant par le désaccord ( $\dot{\alpha} \pi \grave{\partial} \tau \tilde{\eta} \varsigma \delta 1 \alpha \phi \omega \nu i \alpha \varsigma \dot{\alpha} \rho \xi \dot{\alpha} \mu \varepsilon v o l)$ ». Le désaccord en question est présenté en $P H$ II 18 : c'est celui qui oppose ceux qui posent le critère, ceux qui le nient et ceux qui restent en suspens - la question étant de savoir si ces derniers participent du désaccord ou, plus rigoureusement, suivent la conséquence du désaccord entre les deux premiers. Comme l'exposent ensuite $P H$ II 19-20, par un recours aux modes d'Agrippa (en l'occurrence, désaccord, réciprocité et régression à l'infini), ce désaccord ne peut lui-même être tranché que par un critère introuvable sauf par pétition de principe.

18. $M$ VII 261 : « Dès lors que presque la totalité du désaccord ( $\delta$ ( $\alpha \omega \omega \nu i \alpha)$ sur le critère est maintenant sous nos yeux, le moment est venu d'en venir à la réfutation ( $\dot{\alpha} v \tau i p p \eta \sigma ı \zeta)$ et

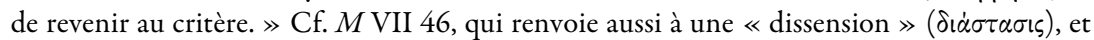

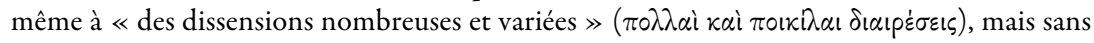
qu'il en soit tiré explicitement un argument philosophique contre le critère. 
69), la troisième partie de sa réfutation n'usant d'aucune formulation particulière. Ces formulations unilatéralement négatives ne trouvent pas leur équivalent dans le passage du Contre les logiciens : la première partie de la réfutation est introduite par l'annonce d'une impasse à venir sur le critère

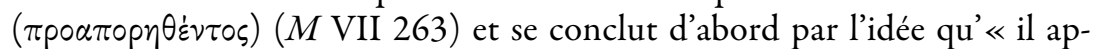
paraît ( $\phi \alpha i v \varepsilon \tau \alpha l)$ que le critère en philosophie est comme introuvable ( $\dot{\omega} s$

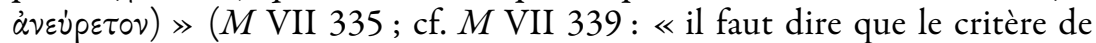

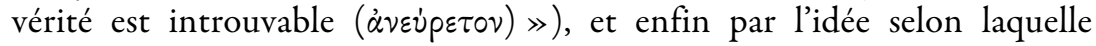
« l'ignorance $(\dot{\alpha} \gamma \nu \omega \sigma i \alpha)$ à propos du critère conduit, du fait de tant d'argu-

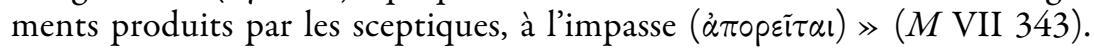
On retrouve la notion d'impasse ( $\dot{\alpha} \pi \circ p i \alpha)$, dont le critère serait rempli ( $\pi \lambda \tilde{n} \rho \varepsilon \varsigma)$, au début de la troisième partie de la réfutation ( $M$ VII 370). En parlant d'impasse ou même du caractère introuvable du critère, Sextus souligne avant tout l'échec subjectif de la recherche, qui conduit à une confession d'ignorance et à la suspension - ce qui contraste fortement avec l'affirmation du caractère inappréhendé et surtout de l'inexistence du critère, qui semble plutôt mettre l'accent sur une caractérisation objective qui traduit davantage un scepticisme de facture nihiliste.

Néanmoins, pour être tout à fait précis, si les deux ouvrages expriment sans doute des nuances différentes, liées peut-être à des contextes d'écriture distincts ${ }^{19}$ ou à des choix argumentatifs volontaires, le type de scepticisme que Sextus Empiricus entend y défendre est explicitement le même. Ainsi, la fin de l'argumentation sur le critère dans les Esquisses ( $P H$ II 79) vient clarifier de la manière la plus nette la position sceptique. Premièrement, « nous ne nous proposons pas d'affirmer que le critère de vérité est inexis-

19. L'hypothèse d'une évolution chronologique de la pensée de Sextus Empiricus entre les deux ouvrages peut être invoquée. Longtemps, la lecture de Janáček 1947 (reprise en 1972), qui voyait en $P H$ un ouvrage antérieur à $M$ VII à partir de considérations uniquement stylistiques, était considérée comme l'orthodoxie en la matière ; mais, sur des considérations stylistiques autant que philosophiques, c'est aujourd'hui la thèse inverse qui tend à s'imposer : voir Brunschwig 1995 (1988), p. 296 n. 1. Cf. Pellegrin 2010, p. 135-139, qui revient sur le débat général à propos de l'évolution chronologique de la pensée de Sextus Empiricus, dont Richard Bett fait une clef de lecture essentielle (voir notamment Bett 2000), même si ses études ont surtout porté sur les comparaisons entre $P H$ et $M$ XI et entre $P H$ et $M$ I-VI. Pour le problème qui nous concerne, si l'on se fie à une première lecture des textes en $P H$ et en $M$ VII, qui se traduit dans les interprétations divergentes de Janáček et Long, on devrait considérer que Sextus a évolué d'une version nihiliste $(P H)$ à une version suspensive ( $M$ VII) du scepticisme sur le critère, ou inversement - ce qui ne laisse pas de poser problème car, selon Bett, la version suspensive du scepticisme est précisément celle qui devrait prévaloir dans le dernier état de la pensée de Sextus exposé en $P H$. Cependant, comme nous le montrons plus loin, il n'y a peut-être pas tant de différence de conception entre $P H$ et $M$ VII, en tout cas sur la question du critère, ce qui permet de pondérer la portée de ces considérations chronologiques. 


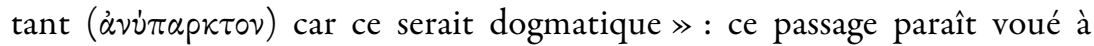
contrer directement l'identification du scepticisme à une négation du

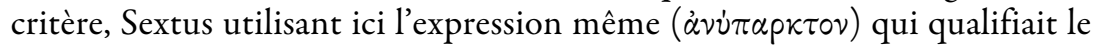
critère un peu plus haut. Deuxièmement, les discours contre le critère exposés par le sceptique sont seulement le pendant des discours pour le critère défendus par les dogmatiques : les premiers ne sont, pour le sceptique, « ni vrais, ni plus convaincants» que les seconds mais ils permettent d'atteindre l'équilibre qui conduit à la mise en suspens ( termes, les formulations nihilistes sont seulement l'une des branches de l'opposition des arguments et n'expriment pas la position du sceptique. Mais une telle conclusion ne semble pas propre aux Esquisses. En effet, on assiste à un mouvement inversé dans la conclusion de l'argumentation sur le critère du Contre les logiciens, Sextus finissant par écrire qu' « il s'en suit

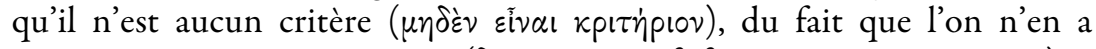

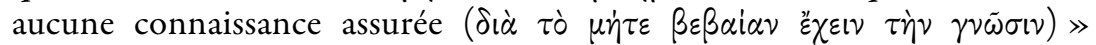
(MVII 439). En concluant de l'incertitude de la connaissance du critère à l'inexistence du critère, Sextus franchit ici la ligne de la confession d'ignorance au nihilisme qu'il s'était gardé de passer tout au long de l'examen mené dans le Contre les logiciens, et il retrouve les formulations des Esquisses. Mais la suite du passage permet là aussi de rétablir un équilibre qui rend les positions de Sextus dans les Esquisses et dans le Contre les logiciens parfaitement concordantes. Ainsi, Sextus présente en $M$ VII 440 une objection dogmatique qui s'appuie sur cette formulation d'allure nihiliste pour contester au sceptique de pouvoir dire que rien n'est critère sans se contredire : en effet, ou bien il dit cela sans critère et il manque de crédit, ou bien il le dit avec un critère et il s'autoréfute. La réponse à cette objection ( $M$ VII 443-444) permet de clarifier l'attitude du sceptique à l'égard du critère. Premièrement ( $M$ VII 443), Sextus reprécise que l'argumentation sceptique consiste à contrebalancer «la conception com-

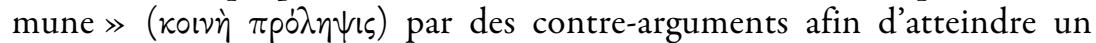
équilibre : ainsi, en l'occurrence, « ce n'est pas pour éliminer le critère (oỉ $k$

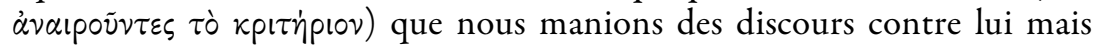
c'est dans l'intention de montrer que le fait qu'il y ait un critère n'est pas totalement garanti ( $\circ \dot{v} \pi \dot{\alpha} \nu \tau \omega \varsigma \pi\llcorner\sigma \tau \dot{\delta} \nu) \gg$, et ce contrairement à ce qui est généralement admis. Deuxièmement ( $M$ VII 444), à la différence des dogmatiques, nous ne donnons pas notre assentiment aux arguments que nous exposons mais nous en restons strictement à l'expression de «l'impression

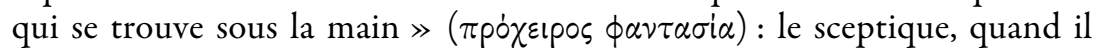
argumente dans un sens, est déjà sous l'effet de l'équilibre des arguments contraires en termes de conviction. Il convient donc de relire tout ce qui est dit sur le critère à cette aune : le sceptique ne nie pas le critère mais il se contente de rendre compte des arguments contre le critère afin de produire 
chez les philosophes la mise en suspens sur la question du critère. Le scepticisme est nécessairement suspensif, sinon il se trahirait: cela vaut pour la discussion du critère comme pour les autres sujets.

Il n'est pas dit que cette ferme mise au point de Sextus Empiricus, qui a le mérite de la cohérence, soit partagée par tous les auteurs sceptiques de l'Antiquité : on peut y voir le résultat ultime d'une histoire de la controverse sur la position des sceptiques sur le critère. Mais on peut également penser que les différentes sources que nous avons rappelées n'avaient pas toujours ou bien la finesse philosophique ou bien la bienveillance nécessaires pour mesurer ce qu'une position nihiliste sur le critère pouvait avoir de contraire à un scepticisme bien compris. Il reste que la mise en suspens sur le critère, si elle ne tranche pas sur son existence ou son inexistence, conduit de fait à ne pas se fonder sur un critère. Un philosophe dogmatique recherche le critère afin de pouvoir fonder dessus son système philosophique dans sa dimension épistémologique, ontologique et morale : dans l'ignorance du critère, un tel système ne saurait prendre place. Inversement, celui qui en reste à une incapacité de se prononcer sur le critère ne saurait prétendre avoir quelque critère que ce soit. C'est pourtant bien ce que certains sceptiques auraient prétendu. C'est cette conciliation contradictoire de la suspension sur le critère et de l'acceptation du critère qu'il convient désormais d'examiner.

\section{Un critère sceptique ?}

De nombreuses sources concordent sur la reconnaissance d'un critère par les sceptiques. Alors qu'il déclarait quelques paragraphes plus haut que les sceptiques «éliminaient» le critère, Diogène Laërce affirme que «le

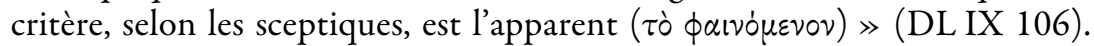
Il n'est pas le seul à l'écrire: on retrouve la même assertion à plusieurs reprises chez Sextus Empiricus (PH I 22; $M$ VII 30), mais aussi dans le Commentaire anonyme sur le Théétète (61.15-22), d'après lequel «selon [Pyrrhon], le critère n'est ni la raison ni l'impression vraie ou convaincante ou appréhensive ni quoique ce soit de tel, mais ce qui lui apparaît main-

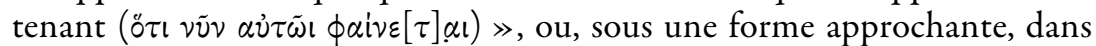
le De Dignoscendis Pulsibus de Galien, qui rapporte que les sceptiques sont ceux « qui posent l'apparaître ( $\tau \dot{\partial} \phi \alpha i v \varepsilon \sigma \theta \alpha \iota ~ \tau \imath \theta \varepsilon \dot{v} \tau \varepsilon \varsigma) »$ (VIII 781). Sans oublier les passages où Sextus Empiricus attribue comme critère à Arcésilas

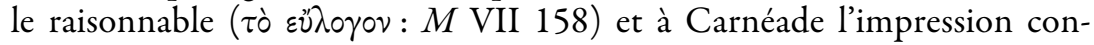

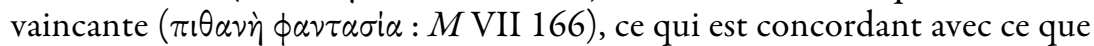
Cicéron en dit dans ses Académiques (II 8, 59, 99, 104). On pourra d'emblée préciser que, contrairement à ce que le Commentaire anonyme pourrait faire penser, les interprètes tendent aujourd'hui à considérer que cette 
conception du critère sceptique ne vient pas de Pyrrhon mais tout au plus de Timon, voire d'Énésidème ${ }^{20}$. Quant à l'idée que le critère d'Arcésilas ait été le raisonnable, l'interprétation la plus courante est aujourd'hui de considérer qu'il s'agit d'une thèse dialectique ou ad hominem, vouée à retourner contre les stoïciens leur propre théorie de l'action droite ${ }^{21}$. Enfin, on peut penser que l'usage de la notion de « critère » par certains sceptiques pour décrire leur position ait répondu au souci de répondre aux canons des écoles philosophiques de la période hellénistique et impériale, mais que le terme est impropre ${ }^{22}$. Diogène Laërce (IX 106) identifie d'ailleurs le critère sceptique qu'est l'apparent au critère d'Épicure (oป̌ $\tau \omega \delta \dot{\varepsilon} \kappa \alpha i$ E $\pi$ ikovpos), dogmatique s'il en est, ce qui pourrait accréditer l'idée qu'une telle formulation crée plus de confusion qu'elle n'exprime la pensée sceptique avec adéquation. Tous ces éléments peuvent laisser penser que la question de la conciliation de la réfutation du critère et de la reconnaissance du critère n'est finalement qu'un faux problème, auquel ne furent pas sérieusement confrontés les sceptiques. Toutefois cette lecture déflationniste de la notion de critère sceptique, qui serait tout au plus un terme abusivement employé par les représentants les plus tardifs de ce courant, s'accorde mal avec l'insistance dont témoignent certains textes pour en faire un enjeu de la définition du scepticisme.

Premièrement, la détermination de la nature du critère sceptique est l'occasion d'une distinction conceptuelle entre deux sens du terme, distinction à laquelle il est fait référence à diverses reprises dans les différents corpus et qui vaudrait ainsi comme une mise au point particulièrement importante. Ce sont les textes de Sextus Empiricus qui sont à cet égard les plus clairs. Celui-ci appelle à bien distinguer entre le critère au

20. Voir Bett 2000, p. 93, et, encore plus nettement, Svavarsson 2010, p. 53-54. Diogène Laërce (IX 106) renvoie la formulation sur le critère sceptique à Énésidème ( $\delta \eta \mu o ́ s ~ \phi \eta \sigma(v)$, ou au moins à Énésidème parmi d'autres comme le kaì peut le faire penser.

21. Voir, récemment, Thorsrud 2010 p. 80 n. 17, ou Striker 2010, p. 199-200, qui maintient donc jusqu'à aujourd'hui cette interprétation désormais classique depuis Couissin. Toutefois, contra Ioppolo 2009, p. 109-130, qui défend l'hypothèse d'un authentique critère arcésilasien.

22. Voir Polito 2007, qui renvoie à tout le début des Esquisses pyrrhoniennes (PH I 5-30) comme à un exercice rhétorique d'apologie - ou d'éloge paradoxal - du scepticisme à l'époque de la reconnaissance officielle des sectes philosophiques par Marc Aurèle; Striker 2010, p. 203 : « the very word "criterion", used no doubt because Sextus' general account of scepticism is organized according to the standard topics in expositions of philosophical doctrine, is somewhat misleading to the extent that it means an instrument of judging: Sextus' text makes it abundantly clear that the Sceptics' way of following appearances does not involve a judgement or decision. 》 
sens dogmatique et le critère en un autre sens, qui peut être accepté par un sceptique.

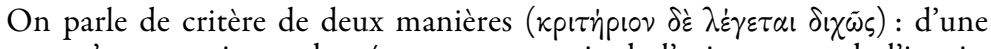
part, c'est ce qui est adopté comme garantie de l'existence et de l'inexistence, à propos duquel nous parlerons dans notre discours réfutatif $(\dot{\varepsilon} \nu \tau \tilde{\omega}$ $\dot{\alpha} \nu \tau \iota p p \eta \tau \iota \kappa \tilde{\omega} \lambda{ }^{\prime} \gamma(\omega)$; d'autre part, c'est ce qui vaut pour agir, auquel nous nous référons dans la vie pour faire telles actions et non telles autres, et à propos duquel nous parlons ici. (PH I 21)

Ce passage des Esquisses pyrrhoniennes, situé dans le chapitre précisément consacré au critère du scepticisme (PH I 21-24), annoncé au début de l'ouvrage (PH I 5), exprime de manière très nette le fait qu'il convient de ne pas confondre le critère qui est l'objet de la réfutation ( $\alpha \tau \tau i p p \eta \sigma \iota \varsigma)$ sceptique $^{23}$ et le critère d'action ( $\tau \circ \tilde{u} \pi \rho \dot{\alpha} \sigma \sigma \varepsilon \iota \nu$ ), dont Sextus dit juste après qu'il est le critère des sceptiques ( $P H$ I 22). On trouve la même distinction au début du livre II des Esquisses (PH II 14), au moment où Sextus aborde justement la réfutation du critère dogmatique, et dans le passage parallèle du Contre les logiciens ( $M$ VII 29), mais également à la fin du premier livre $\mathrm{du}$ Contre les logiciens ( $M$ VII 435), où il est dit que les critères sont « de l'un des deux types suivants ( $\delta$ vยĩ $\theta \dot{\alpha} \tau \varepsilon p o \nu$ ), ou bien c'est quelque chose que l'on conçoit comme utile à la conduite de sa vie, ou bien c'est quelque chose d'utile à la découverte de la vérité dans les choses », sans que soit alors attribuée une dimension dogmatique ou sceptique à aucune des deux acceptions.

Ce renvoi aux deux usages de la notion de critère n'est pas seulement présent chez Sextus : il y est explicitement fait référence dans le chapitre consacré au critère de l'Histoire de la philosophie du Pseudo-Galien (chapitre 12), qui énonce que « le critère est de deux types $(\delta i \tau \tau \dot{o} \nu)$ : d'une part, c'est ce dont nous usons pour vivre; d'autre part, c'est ce qui concerne l'existence et l'inexistence. » Mais il semble également possible de penser que le passage sur le critère pyrrhonien dans le Commentaire anonyme sur le Théétète renvoie aussi à cette dimension pratique du critère, comme peut le faire penser la suite du texte : « sa manière de se conduire ( $\tau \grave{\partial} \delta เ \varepsilon \xi \dot{\alpha} \gamma \varepsilon \iota \nu)$ suit l'impression qui se présente à chaque fois non pas considérée comme vraie

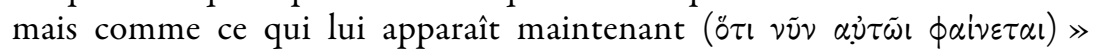
$(61.40-45)^{24}$. On notera toutefois que, contrairement aux passages de

23. Cf. $P H$ II 17 et $M$ VII 261, qui utilisent précisément ce terme.

24. Selon Bett 2000, p. 87, il faudrait également lire le passage de Galien, De dignosc. puls. VIII 781 avec cette dimension pratique, soit la pratique médicale, en l'occurrence. Mais un autre passage de Galien, Esquisse empirique, 82, 23-26, permet d'aller dans ce sens: «Pyrrhon le sceptique, qui, recherchant la vérité et ne la trouvant pas, restait dans 
Sextus et à celui du Pseudo-Galien, il n'est pas fait référence ici à un double sens de la notion de critère mais seulement à un usage de quelque chose comme un critère (la notion elle-même apparaît bien un peu plus haut en 61.15-22) pour conduire sa vie et non pour identifier la vérité. Les passages de Diogène Laërce, selon de nombreux interprètes, seraient à lire de la même manière. Même si la définition générale du critère que Diogène Laërce donne ailleurs est «ce par quoi est connue la vérité des choses » (DL VII 49), ce serait bien au sens d'un critère d'action qu'il conviendrait de comprendre le passage de Diogène Laërce sur le critère des sceptiques (DL IX 106). Il faudrait alors rapprocher cette formulation attribuée à Énésidème de deux autres citations d'Énésidème par Diogène : d'une part, juste un peu plus haut (DL IX 106), il écrit qu' « Énésidème, dans le premier livre de ses Discours pyrrhoniens, énonce que Pyrrhon ne définissait rien de manière dogmatique du fait de la contradiction des discours mais

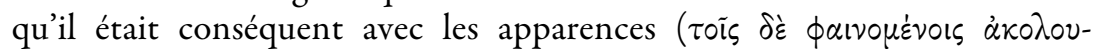
$\theta \varepsilon i v)$ » d'autre part, en DL IX 62, il rapporte qu' « Énésidème énonce que Pyrrhon philosophait selon le discours qui met en suspens mais qu'il n'accomplissait pourtant pas chacune de ses actions ( $\pi \rho \dot{\alpha} \tau \tau \varepsilon เ v)$ de manière non prévoyante », ce qui peut également être rapproché de l'expression du début de IX 62 selon laquelle Pyrrhon «était conséquent dans sa vie »

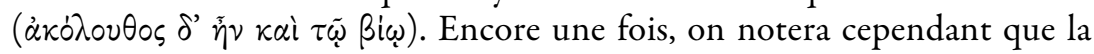
distinction entre deux types de critère, l'un de vérité et l'autre d'action, n'est pas explicitement invoquée, comme c'est clairement le cas chez Sextus Empiricus. Il s'agit plutôt chez Diogène Laërce - et finalement, surtout pour les interprètes contemporains qui semblent largement inspirés de ce que l'on trouve chez Sextus Empiricus - de laisser un espace à la conduite de la vie sceptique, laquelle se serait appuyée sur un critère (comme l'énonce explicitement DL IX 106), mais sans qu'il soit d'ailleurs précisé dans ces passages qu'un tel critère serait de nature différente de celui des dogmatiques. Enfin, comme le laissent penser les passages de Sextus Empiricus sur le critère chez Arcésilas ( $M$ VII 158) et chez Carnéade ( $M$ VII 166) -

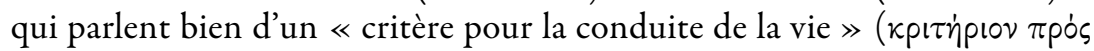

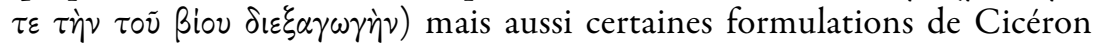
(Ac. II 32, 99-101, 104, 110), il est généralement considéré que les sceptiques académiciens s'appuyaient eux aussi sur une distinction entre critère de vérité, objet de la réfutation sceptique, et critère pratique, sur lequel pouvaient s'appuyer les sceptiques ${ }^{25}$.

l'incertitude sur toutes les choses non manifestes et qui, dans ses actions de tous les jours, suivait l'évidence (sequens evidentia) » (Pellegrin 1998, p. 121).

25. Voir Thorsrud 2010, p. 67-70 (Arcésilas) et p. 72-74 (Carnéade). Sur les passages chez Sextus, voir Ioppolo 2009, p. 109-130 (Arcésilas) et p. 141-160 (Carnéade). Sur les 
Cette série de remarques permet ainsi de proposer l'hypothèse suivante : la notion de critère sceptique, quand elle est explicitement nommée, comme c'est le cas chez tous les auteurs que nous venons de mentionner, serait toujours liée à la question de la conduite de la vie, même si elle ne s'énonce pas forcément comme un type de critère à part entière critère d'action distinct du critère de vérité - ainsi qu'on peut le trouver chez Sextus Empiricus ou le Pseudo-Galien. Même s'il est toujours difficile de faire des conjectures en la matière, mais compte tenu de la chronologie que l'on peut reconstituer entre les textes, il semble possible de supposer que la thématique du critère sceptique s'est progressivement imposée comme une alternative à part entière au critère dogmatique. Cela commencerait dans le scepticisme académique tel que Cicéron en rend compte dans les Académiques, où la notion de critère apparaît en elle-même moins centrale que celle plus générale d'un type d'impression permettant d'agir sans appréhension ou sans vérité : l'expression de «critère pratique », utilisée par certains interprètes récents pour décrire la position d'Arcésilas et de Carnéade, apparaît dès lors sans doute légèrement anachronique, et l'on pourra considérer que Sextus Empiricus fait entrer les deux auteurs dans un cadre conceptuel (celui de la distinction entre les deux critères) qui est le sien mais qui n'était pas le leur ${ }^{26}$. Énésidème, critique de l'académisme auquel il reproche son dogmatisme, aurait peut-être donné à la notion de critère sceptique une nouvelle dimension, en passant par une réinterprétation de Pyrrhon : c'est ce dont pourraient témoigner les passages du Commentaire anonyme du Théétète et de Diogène Laërce, où le terme de


$\phi \alpha \iota v o ́ \mu \varepsilon v o v)$ valant comme alternative aux différentes formes de critère dogmatique et pouvant servir de base pour conduire sa vie au moment même où toutes les thèses dogmatiques sont réfutées. Là encore, on ne peut dire à proprement parler que le critère sceptique soit conçu comme un critère

passages chez Cicéron, voir Brittain 2006, p. XXIV et surtout XXVI-XXVII, où il définit le «practical criterion 》 comme « a truth-indifferent mechanism for rational action » qui permet « to act both in ordinary life and in the course of philosophical arguments 》: il renvoie à cette notion de critère pratique à de nombreuses reprises dans les notes de sa traduction (n. 42 p. $20-21$; n. 46 p. 22 ; n. 85 p. 38 ; n. 147 et 148 p. 58 ; n. 152 p. 60 ; n. 163 p. 63), même si le seul passage qui semble mentionner explicitement l'idée d'un tel «critère »se trouve uniquement en II 32 : «their guiding rule [ $\kappa \alpha \nu \omega \nu$ ?] both for conducting their lives and in investigation and argument $\gg$ (Brittain 2006, p. 21).

26. Voir Bett 1989, p. 82, qui considère que la distinction entre les deux formes de critère est plutôt une réécriture de Sextus, contra Ioppolo 2009, n. 136 p. 175, qui renvoie à Plutarque, Adv. Colotem 26, 1122 B-D ; Contradiction des Stoïciens 47, 1057 A et à Cicéron, Lucullus 32, 99, 104, 110. On notera que, à l'exception peut-être de Cicéron, Lucullus 32, aucun des passages cités par Ioppolo ne mentionne la notion de « critère ». 
pratique par distinction avec le critère de vérité des dogmatiques: mais cette forme de présentation affleure presque, et certains interprètes un peu hâtifs l'ont clairement inféré27. Enfin, avec Sextus Empiricus et le PseudoGalien, puisant à une source commune - à moins que le Pseudo-Galien ne s'inspire de Sextus lui-même, ou inversement -, la distinction entre les deux formes de critère est explicite et permet, chez Sextus, de faire la part entre un critère sceptique, identifié à un critère d'action, et un critère de vérité, qui ne saurait être que dogmatique et donc inappréhendé. Si Sextus Empiricus a donc sans doute durci la conception sceptique du critère, au risque d'ailleurs de tomber dans l'impasse de l'usage d'une notion hautement dogmatique dans un contexte sceptique, il semble se placer dans la parfaite continuité d'un mouvement historique qui va des académiciens aux néopyrrhoniens. Cela constitue un véritable tour de force, dès lors que la notion de critère est fortement teintée de dogmatisme et qu'une telle réappropriation pleinement assumée, ce qui n'était peut-être pas le cas chez Arcésilas et Carnéade voire Enésidème, est lourde de dangers et de possibles contradictions. Nous verrons plus loin si Sextus Empiricus en a bien consciemment mesuré tous les risques philosophiques. Mais il apparaît en tout cas manifeste que la position d'un critère sceptique est bien plus qu'un abus de langage ou la lubie d'un auteur tardif.

Deuxièmement, la notion de critère sceptique, conçu comme critère d'action, apparaît non seulement comme un élément central de la distinction entre scepticisme et dogmatisme mais également comme une manière, interne à l'histoire du scepticisme, de différencier scepticisme et pseudo-scepticisme. Cette question traverse implicitement les Académiques de Cicéron, ouvrage dans lequel la position académicienne de Carnéade sur le critère est interprétée tantôt en des termes nihilistes (par Lucullus s'appuyant sur Antiochus d'Ascalon, $A c$. II 32-34) - c'est-à-dire que le critère d'action est rendu impossible par la réfutation du critère de vérité -, tantôt en des termes sceptiques (par Cicéron citant Clitomaque de Carthage, $A c$.

27. Voir Hankinson 2010, p. 106 : le critère de DL IX 106 « is the practical criterion, not any "criterion of truth" ». Bett 2000, p. 84-93, rapproche l'ensemble de ces textes (Sextus Empiricus, Diogène Laërce, Galien, Commentaire anonyme) pour conclure au fait que, si la notion de critère d'action n'est sans doute pas attribuable à Pyrrhon lui-même, il est vraisemblable qu'elle ait été très tôt sous-jacente à la réflexion sceptique pyrrhonienne. Bett considère même, en renvoyant à Aristote, Métaphysique $\Gamma 1008 \mathrm{~b} 20$, que l'exemple du miel, que l'on trouve en DL IX 105 comme en $P H$ I 19-20, aurait une teinte « pratique » (p. 88). Cette conception d'un critère sceptique comme critère d'action, reprise par Énésidème puis par Sextus Empiricus, n'aurait cependant pas eu le même fondement philosophique, selon Bett : sur un fonds métaphysique chez Pyrrhon, elle s'appuierait sur une pensée métaphysico-épistémologique (relativiste) chez Énésidème, et finalement sur une pensée strictement épistémologique chez Sextus (p. 217 et 233-236). 
II 99-105) - c'est-à-dire que la position d'un critère d'action est compatible avec la réfutation du critère de vérité -, tantôt en des termes probabilistes (par Cicéron s'inspirant sans doute de Philon de Larissa, Ac. II 110-111) c'est-à-dire que le critère pratique peut servir comme une forme de critère de véritée ${ }^{28}$. Le discours de Cicéron se conclut de fait sur des assertions surprenantes, si l'on considère que les académiciens renoncent au critère de vérité pour adopter seulement un critère pratique.

$<$ La réfutation d'Antiochus $>$ tomberait juste si nous, académiciens, nous débarrassions tout uniment de la vérité. Cependant, ce n'est pas ce que nous faisons, puisque nous discernons [ кpivelv ?] autant de choses vraies que de choses fausses. Mais notre acte de discernement [kpiбis ?] est une forme d'approbation; nous n'y trouvons aucune marque d'appréhension. (Ac. II 111)

Ce que ce passage met en lumière est que pour Cicéron, s'inspirant davantage de Philon que de Clitomaque, la frontière entre un dogmatique et un sceptique serait finalement moins la différence entre critère de vérité et critère d'action que celle entre critère de vérité qui va jusqu'à l'appréhension, c'est-à-dire qui se prononce sur les choses non manifestes et qui donne son assentiment, et critère de vérité qui ne va pas jusqu'à l'appréhension et se contente d'une approbation ${ }^{29}$. C'est précisément cette thèse qui fait que, d'après Photius (Bibliothéque, 212, 170a), Énésidème conteste aux académiciens d'être des sceptiques - même si l'argument d'Énésidème rapporté par Photius néglige la distinction fondamentale entre «vrai » $(\dot{\alpha} \lambda \eta \theta \dot{\varepsilon} \zeta)$ et « appréhendé » ( $\kappa \alpha \tau \alpha \lambda \eta \pi \tau \dot{\delta} \nu)$, et tend à faire penser qu'un sceptique véritable est celui qui n'a pas de critère du tout.

Chez Sextus Empiricus, en revanche, il est intéressant de constater que c'est notamment autour de la notion de critère sceptique, c'est-à-dire du critère d'action, que se joue la différenciation entre pyrrhoniens (authentiquement sceptiques) et académiciens (finalement dogmatiques). Comme nous l'avons vu (PH I 21), Sextus distingue le critère dogmatique et le critère sceptique en ce que le premier est « ce qui est adopté comme ga-

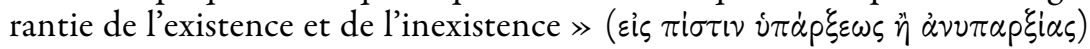
des choses, alors que le second est « ce qui vaut pour agir, auquel nous nous

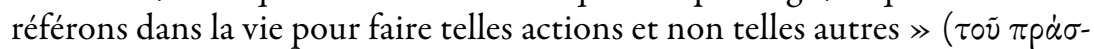

28. Voir Brittain 2006, p. XIX-XXI.

29. Cf. $A c$. II 119 : « Peut-être que ces doctrines sont vraies - note que j'admets ici qu'il puisse y avoir des vérités - ; mais je n'accepte toujours pas qu'elles soient appréhendées. » Sur l'idée que les académiciens ne disent pas que tout est non manifeste ( $\alpha \delta \eta \lambda o v)$ mais que tout est non appréhendé ( $\dot{\alpha} \kappa \alpha \tau \dot{\alpha} \lambda \eta \pi \tau \sigma \nu)$, voir $A c$. II 32, Eusèbe, Prép. év. XIV 7, $\S 15$. Cette doctrine semble davantage celle de Philon (la prétendue « quatrième » Académie) que celle de Carnéade : voir Brittain 2006, p. XXIX. 



cription du critère sceptique est développée quelques lignes plus loin.

Ainsi, nous référant aux apparences, nous vivons sans opinion selon l'observation de la vie ( $\kappa \alpha \tau \dot{\alpha} \tau \dot{\eta} \nu \beta \iota \omega \tau \kappa \grave{\nu} \nu \tau \dot{p} p \eta \sigma \nu)$, puisque nous ne pouvons être totalement inactifs. Et cette observation de la vie semble résider dans quatre parties, qui sont les indications de la nature, la nécessité des affections, le suivi des lois et des coutumes et l'enseignement des arts. (PH I 23)

Or, quand, à la fin du premier livre des Esquisses, Sextus expose ce qui différencie les académiciens des sceptiques, il écrit (PH I 226) que, pour les premiers, « il est convaincant que ce qu'ils disent être bon est réellement le cas $(\dot{v} \pi \dot{\alpha} \rho \chi \varepsilon \imath \nu)$ plutôt que le contraire $\gg$, ce qui leur fait finalement adopter une forme de critère de vérité, alors que les seconds se contentent de « suivre sans opinion la vie $(\tau \tilde{\omega} \beta \hat{\beta} i \omega)$ afin de ne pas être inactifs », ce qui est quasiment une citation mot pour mot de l'observation vitale qui vient développer la notion de critère d'action. De même, Sextus écrit plus loin (PH I 231) que les académiciens « ont recours au convaincant dans la vie », tandis que les sceptiques «vivent sans opinion en suivant les lois et coutumes et les affections naturelles » - autre référence explicite à l'observation vitale qui exprime le critère d'action, alors même que le critère académicien, même dans la vie, renverrait ultimement à la vérité. Ce glissement du critère d'action au critère de vérité chez les héritiers de Carnéade, selon Sextus, s'opère au sein même de sa longue présentation de la conception du convaincant comme critère dans le Contre les logiciens ( $M$ VII 166189). Ainsi, après avoir clairement attribué à Carnéade la réfutation du critère de vérité et l'adoption d'un critère «pour la conduite de la vie » (MVII 166), Sextus écrit que «l'impression qui apparaît vraie et qui est

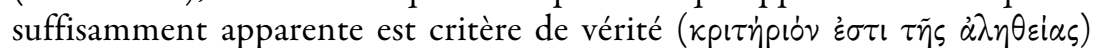
selon Carnéade et les siens » ( $M$ VII 173) ou que « l'Académicien procède

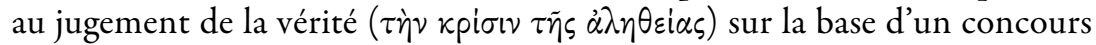
d'impressions » ( $M$ VII 179). Certains interprètes y voient une déformation, volontaire ou involontaire, de la doctrine de Carnéade, laquelle rendrait en elle-même impossible l'adoption d'un critère de vérité et, inversement, nécessaire l'adoption d'un critère d'action ${ }^{30}$. Il n'empêche que c'est

30. Voir Ioppolo 2009, p. 148-152, 157. Contra Thorsrud 2010 p. 73-74 et 78, qui concède que la notion même de convaincant implique presque nécessairement un rapport au vrai, et que la polémique entre Clitomaque (le convaincant n'est pas critère de vérité mais tout au plus critère d'action) et Philon (le convaincant est non seulement critère d'action mais aussi critère de vérité), dont les Académiques de Cicéron sont le reflet, illustre la difficulté qu'ont eue les disciples de Carnéade pour cerner la position exacte de leur maître sur ce point. 
bien autour de la notion de critère sceptique, conçu comme critère strictement pratique, que s'opère, chez Sextus, la distinction entre sceptiques et non-sceptiques. On pourra également renvoyer à cet égard à la première partie de la réfutation de la thèse selon laquelle le convaincant est critère, réfutation qui vient clore le premier livre du Contre les logiciens (MVII 435-437). Commençant par reprendre la distinction entre les deux critères, celui qui est utile « à la conduite de la vie » et celui qui sert « à la découverte de la vérité dans les choses », Sextus récuse l'idée que l'impression convaincante puisse être critère d'action dans la mesure où « aucune de ces impressions convaincantes ne peut servir en propre ( $\left.\kappa \alpha \tau^{\prime} i \delta i \alpha \nu\right)$ aux conduites de la vie, mais chaque conduite ${ }^{31}$ a besoin de l'observation

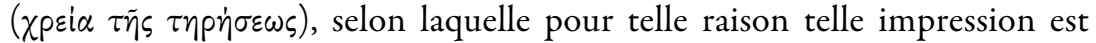
convaincante et pour telle raison telle autre est suffisamment vérifiée et incontournable $\gg$. L'argument est généralement compris comme une manière, de la part de Sextus, de renvoyer ici l'impression convaincante à un jugement préalable, assuré par l'observation, c'est-à-dire à une forme de critère de vérité ${ }^{32}$. Or, on peut y voir plutôt un renvoi au fait que, en

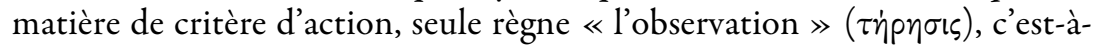
dire précisément la notion qui décrit le critère des pyrrhoniens selon $P H \mathrm{I}$ 23-24 et les passages parallèles ( $P H$ II 254, PH III 235, $M$ XI 165, $M$ V 2, cf. $P H$ I 226, $P H$ I 231). Dans un cas comme dans l'autre, les académiciens se voient contester l'adoption d'un authentique critère d'action : ils useraient, même dans la vie, d'un critère de vérité et seraient, de ce fait, des dogmatiques qui s'ignorent ${ }^{33}$.

Troisièmement, la notion de critère sceptique joue un rôle central dans la réponse aux objections dogmatiques contre le scepticisme. En effet, selon une objection classique au scepticisme, le sceptique se trouve face à deux branches également inacceptables d'une alternative: ou bien le sceptique est incapable de faire un choix et donc ne peut plus rien faire, ou bien le

31. Le texte porte $\dot{\varepsilon} \alpha \dot{\alpha} \sigma \tau \eta$, qui renvoie forcément à un féminin pluriel précédent, c'est-àdire soit aux impressions $(\phi \alpha \nu \tau \alpha \sigma i \tilde{\omega} \nu)$ soit aux conduites $(\delta เ \varepsilon \xi \alpha \gamma \omega \gamma \dot{\alpha} \varsigma)$. Les traducteurs choisissent généralement la première solution, en effaçant le pluriel de $\delta\llcorner\xi \xi \alpha \gamma \omega \gamma \dot{\alpha} \varsigma$ (Bury, II, p. 235 ; Bett 2005, p. 86-87) ; pourtant, l'étrangeté du pluriel $\delta 1 \varepsilon \xi \alpha \gamma \omega \gamma \dot{\alpha} \varsigma$ peut précisément faire pencher pour la deuxième solution. C'est surtout le sens même du passage qui fait choisir cette deuxième option, car ce ne sont pas les impressions qui ont besoin d'un critère dans cet argument qui traite du critère d'action, mais bien les conduites.

32. Voir Ioppolo 2009, p. 174 : « Infatti prima di agire è necessario sottoporre ciascuna di esse a una verifica ossertiva e questo significa che bisogna avere un criterio di verità in base a cui giudicare la loro probabilità. »Cf. Brittain 2006, p. XXIX et n. 49.

33. Pour Ioppolo 2009, p. 173-185, Sextus Empiricus attribuerait ici à Carnéade ce qui vaut tout au plus pour Philon : il serait ici influencé par sa source, soit, d'après Ioppolo suivant Janáček, Énésidème (p. 176-178). 
sceptique fait un choix et tombe alors dans le dogmatisme. C'est cette impasse que Diogène exprime dans le paragraphe qui suit celui où il rapporte que les sceptiques ont un critère.

Contre ce critère des apparences, les dogmatiques disent que, quand à partir des mêmes choses se présentent des impressions différentes - comme à partir d'une tour ronde ou carrée -, le sceptique, s'il ne donne sa préférence à aucune, n'agira pas, tandis que, s'il est conséquent avec l'une d'entre elles, il ne donnera plus une force égale aux phénomènes. (DL IX 107)

Dans ce passage, l'argument du conflit des apparences, avec le fameux exemple de la tour, est renvoyé contre les sceptiques : alors que les sceptiques font valoir aux dogmatiques qu'il est impossible de trancher entre les apparences contradictoires d'une même chose et qu'il est donc impossible de dire ce qu'est réellement la chose, les dogmatiques répliquent que, ou bien ce conflit est effectivement indécidable, et toute action deviendra ellemême impossible, ou bien on tranchera ce conflit, et on devra donner une valeur de vérité à l'une des apparences ${ }^{34}$.

Avant d'en venir à la réponse sceptique à cette objection telle que rapportée par Diogène, on notera le caractère ambigu de l'expression de

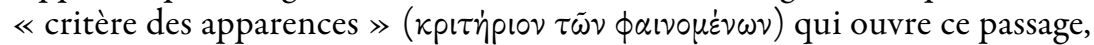
et ce sous deux aspects. Premièrement, un «critère des apparences » peut être compris comme le critère que sont les apparences - ce que semblait poser DL IX 106 - ou bien comme un critère pour distinguer entre les apparences - ce qui semble plutôt l'objet de DL IX 107. Deuxièmement, estce que le «critère des apparences » est finalement identifiable à n'importe quel «critère des choses » (кpıтท่pıov $\tau \tilde{\omega} \nu \pi \rho \alpha \gamma \mu \dot{\alpha} \tau \omega \nu)$, selon la manière classique de parler du critère que l'on trouve par exemple en DL VII 46 et qui semble celle qui permet à Diogène d'identifier le critère sceptique et le critère épicurien en DL IX 106, ou bien est-ce qu'il désigne un acte de jugement qui se situe à un autre niveau, celui des apparences comme distinctes des choses mêmes, comme semble clairement l'indiquer le même Diogène quand il rapporte l'effort sceptique pour distinguer entre l'apparaître, qu'il pose, et l'être, qu'il ne pose ni ne nie (DL IX 105-106) ? Cette double ambiguïté conduisit sans doute à certains malentendus sur la

34. Cf. Vogt 2010 : « But how can adherence to appearances make the sceptic perform some actions and not others? Would it not seem, in particular from the sceptic's own point of view, that there are regularly several conflicting appearances? » (P. 172.) Vogt 2010 parle alors de l'accusation de paralysie («paralysis charge ») : « There is no way to settle on any one of several, mutually incompatible actions available at a given time. » (P. 166.) Le conflit des apparences renvoie en fait à une double paralysie: paralysie devant les apparences contradictoires, mais paralysie aussi liée au fait de devoir faire un choix qui conduirait au dogmatisme. 
nature du critère sceptique, mais elle est également révélatrice de réelles difficultés pour comprendre cette conception. La réplique sceptique rapportée par Diogène ne permet d'ailleurs pas de lever toutes ces difficultés. Il écrit en effet que « contre eux, les sceptiques disent que "quand se présentent des impressions différentes, nous dirons que chacune d'entre elles apparaît" ; et c'est en ce qu'elles apparaissent qu'ils disent poser les apparences » (DL IX 107). D'après ce texte, dont la syntaxe est assez peu claire, la réponse à l'objection résiderait dans le simple rappel du phénoménisme sceptique : le sceptique s'en tient à l'apparence ${ }^{35}$. Et donc, par rapport à la double ambiguïté que nous venons de mettre en évidence, le sceptique pose bien que le critère des apparences n'est pas un critère de distinction entre les apparences (« nous dirons que chacune d'entre elles apparaît ») et que le critère des apparences n'est pas un critère des choses («c'est en ce qu'elles apparaissent qu'ils disent poser les apparences »). En quoi cette réplique répond-elle à l'objection dogmatique ? On ne voit pas bien, sauf à compléter la réponse par l'idée que l'apparence en tant qu'apparence a une force propre qui impose de la suivre malgré le fait que toutes les apparences ont une force égale (i $\sigma \circ \sigma \theta \varepsilon v \varepsilon i \alpha)$ - principe sceptique rappelé ironiquement dans l'objection dogmatique de DL IX 106 -, comme le suggère la citation de Timon selon lequel «l'apparent impose sa force $(\sigma \theta \dot{\varepsilon} v \varepsilon l)$ partout où il passe », citée plus haut par Diogène (DL IX 105), et qui est de fait un vers auquel il est fait mention dès qu'il s'agit du critère sceptique (Sextus Empiricus, $M$ VII 30 ; Galien, De dignosc. puls. VIII 781). L'argument consisterait à dire que l'alternative entre paralysie et choix dogmatique n'est opérante que parce que les dogmatiques ne font pas la distinction entre critère de vérité et critère sans vérité ou critère sceptique: dès lors que cette distinction est posée, un choix sceptique est tout à fait concevable.

Comme nous le voyons, le phénoménisme sceptique (le critère est l'apparent en tant qu'apparent) ne suffit pas pour répondre tout à fait à l'objection, mais il doit s'accompagner d'une forme de déterminisme (l'apparent est ce qui s'impose nécessairement à nous) dont nous trouvons la formulation à l'ultime fin du passage de Diogène.

Ce n'est pas pour autant que cela dépend de nous ( $\pi \varepsilon p \grave{i} \dot{\eta} \mu \tilde{\alpha} \varsigma)$ que nous choisirons ceci ou refuserons cela : mais c'est que nous ne pouvons refuser

35. Cf. Plutarque, Contre Colotès, $1122 \mathrm{E}$, qui rapporte une objection un peu semblable adressée à Arcésilas, et sa réponse phénoméniste : « mais comment celui qui reste en suspens court-il au bain et non à la colline, prend-il la porte et non le mur s'il veut se rendre au

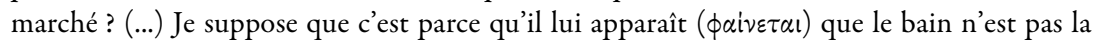
colline mais le bain, la porte pas le mur mais la porte, et de même pour chacune des autres choses. » 
tout ce qui ne dépend pas de nous mais de la nécessité ( $\left.\kappa \alpha \tau^{\prime} \dot{\alpha} \nu \dot{\alpha} \gamma \kappa \eta \nu\right)$, comme la faim, la soif et la douleur - car il n'est pas du ressort de la raison de s'en débarrasser. (DL IX 108.)

Selon les textes sur le critère sceptique, nous trouvons ainsi exprimée tantôt la ligne phénoméniste, tantôt la ligne déterministe, mais c'est bien parce qu'il s'impose avec nécessité que l'apparent est critère ${ }^{36}$. Mais, s'agit-il proprement d'un critère, c'est-à-dire de la base nous permettant de juger et faire un choix, ou ne serait-ce pas seulement une explication a posteriori des choix du sceptique ${ }^{37}$ ? De ce point de vue, l'accusation de paralysie, telle que formulée en DL IX 107, ne sera plus une accusation d'inactivité ou réduction à l'état de plante - car il ne sera pas contesté que le sceptique est actif -, mais une accusation d'inaction ou réduction à l'état d'animal dépourvu de choix rationnel ${ }^{38}$.

À cet égard, la réponse que Sextus propose à l'alternative de l'objection dogmatique entre paralysie et choix dogmatique ( $M$ XI 162-166) differe sensiblement de la réponse rapportée par Diogène. L'objection dogmatique est elle-même exposée différemment : la première branche de l'alternative, la réducation à la paralysie, renvoie moins au conflit des apparences qu'au fait que « la vie (Bios) réside dans des choix et des refus » dont le scepticisme rendrait incapable ( $M$ XI 162-163); quant à la seconde branche de l'alternative, la réduction au dogmatisme, elle s'appuie sur un dilemme moral proprement humain, par l'exemple du tyran qui impose de choisir entre la perte de sa vie et l'accomplissement d'actes innommables ( $M$ XI 164). Le problème posé par les dogmatiques aux sceptiques dans ce passage est donc moins celui de la possibilité épistémologique d'un critère sceptique (comment concilier conflit des apparences et position de l'apparent comme critère ?) que celui de la possibilité vitale du critère sceptique (comment concilier réfutation du bien et du mal et position du critère sceptique comme critère d'action ?). Davantage, on pourra penser que la

36. Pour la ligne phénoméniste, voir $P H$ I 19-22, DL IX 105-107 ; cf. PH I 163, $M$ XI 118 début. Pour la ligne déterministe, voir aussi $P H$ I 19-22, DL IX 108, mais surtout $M$ XI 118 fin, 141-143, 147-148, 156, 159. Voir Annas 1986, p. $19:$ : He will act, both because some motives to action are unavoidable, and because in all matters something will always appear to be the better course, and will work on him accordingly. »

37. Voir Vogt 2010, p. 167 : « the sceptic can formulate a so-called practical criterion, an explanation of what it is that makes the sceptic active rather than inactive, performing one action rather than another. . Cf. p. $171:$ : They can readily explain why the sceptic adheres to this impression rather than to that one (i.e. the sceptic is not, by letting go of assent, but retaining impression and impulse, faced with an impossible stalemate among multiple conflicting impressions). 》

38. Voir Vogt 2010, p. 166 (pour les catégories d'objection), et surtout Perin 2010, chapitre 4, p. 90-113 (sur le critère sceptique et le passage de l'activité à l'action). 
résolution du problème de la possibilité épistémologique du critère sceptique par le phénoménisme et le déterminisme conduit à un approfondissement lié au problème de la possibilité vitale du critère sceptique : l'insistance de Sextus sur la notion de critère pratique ( $P H$ I 21, $P H$ II 14, $M$ VII 29), qui est tout au plus seulement implicite chez Diogène, pourrait ainsi marquer ce déplacement d'accent ${ }^{39}$. La réplique sceptique en $M$ XI 165-166 ne renvoie en tout cas plus du tout au phénoménisme que l'on trouve dans le passage parallèle de Diogène (DL IX 107), voire au déterminisme qui peut lui être lié, mais seulement à la notion d'« observation de

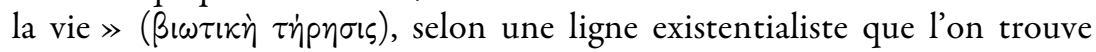
ailleurs chez Sextus mais aussi ponctuellement chez Diogène ${ }^{40}$.

Ainsi, si la notion de critère sceptique n'a pas toujours été thématisée comme telle dans l'histoire du scepticisme antique, elle semble bien plus que la simple reprise maladroite d'une notion dogmatique de premier ordre. Défendre la thèse d'un authentique critère sceptique s'avère un moyen essentiel pour clarifier la nature même du scepticisme. En effet, premièrement, le critère sceptique n'est pas un critère de vérité mais un critère d'action sans vérité ; deuxièmement, toute forme de scepticisme qui renverrait, explicitement ou implicitement, le critère d'action à un critère de vérité se changerait de ce fait même en dogmatisme; troisièmement, la notion de critère sceptique est explicitement conçue comme suffisamment robuste, que ce soit par son renvoi à une explication par la nécessité des apparences ou par la mise en évidence de motifs fondés sur l'observation de

39. Pour Vogt 2010, p. 173-175, c'est ce passage des apparences au suivi de la vie ordinaire (selon le modèle de $P H$ I 21-24) qui permet véritablement au critère sceptique d'être un moyen pour décider ce qu'il faut choisir dans telle ou telle circonstance, et donc de répondre aux objections dogmatiques.

40. MXI 165 : « le sceptique ne vit pas selon la raison philosophique, car de ce point de

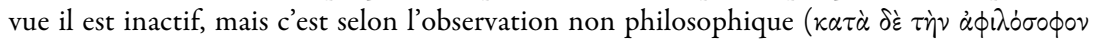
$\tau \dot{\eta} p \eta \sigma(v)$ qu'il peut choisir ou refuser »; M XI 166: « contraint par un tyran à accomplir des actions indicibles, le cas échéant il choisira ou refusera sur la base de la préconception conforme aux lois et coutumes de ses pères. » Ces textes sont à rapprocher de PH I 23-24 et de tous les autres passages où Sextus renvoie, implicitement ou explicitement, à l'observation de la vie. $C f$. DL IX 108 fin : « Et quand les dogmatiques disent que le sceptique pourra vivre mais sans refuser, $s$ 'il en reçoit l'ordre, de dépecer son père, les sceptiques répondent qu'il pourra vivre en se tenant à l'écart des recherches dogmatiques mais pas des

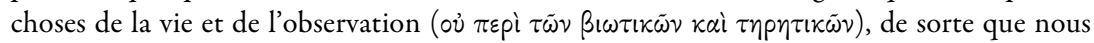
choisissons et refusons selon l'habitude ( $\kappa \alpha \tau \dot{\alpha} \tau \dot{\eta} \nu ~ \sigma u v \dot{\eta} \theta \varepsilon i \alpha \nu)$ et nous faisons usage des lois. » Un texte de Sextus (PH III 235) articule assez clairement ligne phénoméniste et ligne existentialiste: «le sceptique, constatant tant d'irrégularité dans les choses $(\dot{\alpha} \nu \omega \mu \alpha \lambda i \alpha \nu \tau \tilde{\omega} \nu$ $\pi p \alpha \gamma \mu \dot{\alpha} \tau \omega \nu$ ), reste en suspens sur le bien, le mal ou, plus généralement, ce qu'il faut faire par nature et en cela il se départ de la précipitation dogmatique, mais il suit sans opinion l'ob-

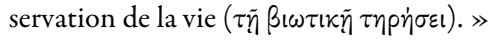


la vie, pour résister aux objections dogmatiques. Mais si le sceptique a un critère d'un genre radicalement différent de celui des dogmatiques, ne peuton sur cette base faire du scepticisme une pensée alternative à la philosophie dogmatique, et non seulement un exercice négatif de réfutation du dogmatisme ? C'est cette question que nous allons maintenant aborder, en approfondissant la compréhension, par une analyse précise des textes, de ce qui distingue le critère sceptique du critère dogmatique et de ce qui en constitue le noyau, soit cette dimension pratique ou vitale.

\section{III - Un critère de vie}

Nous avons déjà énoncé de manière générale ce qui distingue le critère dogmatique du critère utilisé par un sceptique : le premier est un critère de vérité, tandis que le second est un critère d'action. Il reste toutefois à préciser ce qui constitue le fond de cette différence afin de ne pas commettre de malentendus et de mesurer toute la portée de cette distinction. D'après le passage de l'Histoire de la philosophie du Pseudo-Galien (chapitre $12)$, il y aurait à faire une distinction entre un critère pour vivre ( $\beta$ เoṽ) et

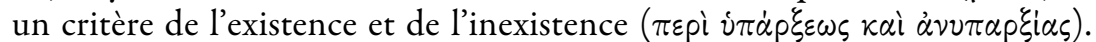
La formulation ramassée du deuxième livre des Esquisses pyrrhoniennes de Sextus Empiricus (PH II 14) rapporte une différenciation fondée exactement sur les mêmes notions, qui apparaissent ainsi comme tout à fait structurantes. Les variations de formulation du passage du premier livre des $E s$ quisses (PH I 21) ne semblent à cet égard pas significatives : gardant la notion d'existence et d'inexistence, d'une part, Sextus parle alors plutôt, d'autre part, d'un critère pour agir ( $\tau 0 \tilde{\nu} \pi \rho \dot{\alpha} \sigma \sigma \varepsilon \iota v)$, même s'il précise aussitôt que ce critère est celui par lequel « dans la vie ( $\kappa \alpha \tau \dot{\alpha} \tau \dot{o} \nu$ ßiov) » nous agissons de telle ou telle manière. Ce noyau de la distinction, entre critère pour vivre ou agir, d'une part, et critère de l'existence et de l'inexistence, d'autre part, se trouve exprimé d'une manière encore différente dans le passage du début du Contre les logiciens ( $M$ VII 29-30), Sextus parlant alors, d'une part, d'un critère pour faire ou ne pas faire ( $\tau \dot{\alpha} \mu \dot{\varepsilon} \nu \pi 010 \tilde{\nu} \mu \varepsilon \nu \tau \dot{\alpha} \delta \dot{\varepsilon}$ ov̉ $\delta \mu \tilde{\omega} s)$

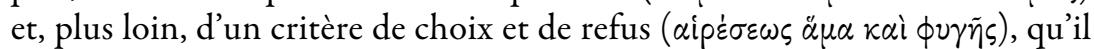
différencie dans ce passage non seulement d'un critère permettant de dire

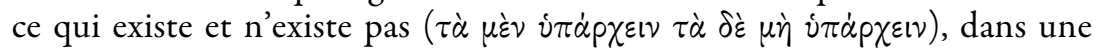
formulation proche de celle des autres passages, mais d'un critère per-

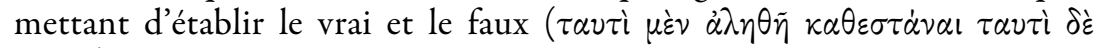
$\psi \varepsilon v \delta \tilde{\eta})^{41}$. Ces variations ne sont pas là pour nous surprendre si l'on considère, d'une part, que la vie est précisément décrite, par exemple en $M \mathrm{XI}$ 162, comme ce qui réside dans des choix et des refus et, d'autre part, que le

41. Voir Corti 2009, p. 75-76. 
vrai est généralement identifié chez Sextus, s’appuyant sur les définitions dogmatiques courantes ( $M$ VIII 9-10), à ce qui existe ou à ce qui dit ce qui existe. Enfin, les derniers passages qui s'appuient sur la distinction mettent

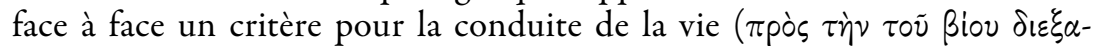
$\gamma \omega \gamma \dot{\eta} v)(M$ VII 158, 166, 435), parfois développé en critère de choix et de refus ou en général d'action ( $M$ VII 158), d'une part, et un critère pour la

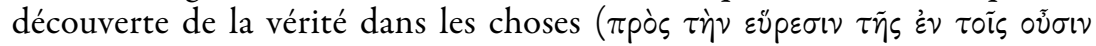
$\dot{\alpha} \lambda \eta \theta \varepsilon i \alpha \varsigma: M$ VII 435), d'autre part.

Cet ensemble de passages permet de poser ce qui constitue la structure générale de la dichotomie entre les deux critères: un critère de vie ou d'action, c'est-à-dire permettant de faire des choix, choix qui sont constitutifs d'une certaine conduite, d'une part, un critère d'existence ou de vérité, c'est-à-dire permettant de dire ce que sont les choses, d'autre part. Exprimée en ces termes, la distinction pourrait laisser penser qu'il s'agit là de deux domaines parfaitement séparés : le domaine de la vie humaine et de la manière de guider sa vie, domaine de l'éthique ou de la morale, d'une part, et le domaine de la recherche de la vérité, domaine de la théorie ou de la connaissance, d'autre part. Or, il apparaît d'emblée qu'une telle interprétation de la séparation des domaines est erronée et crée des confusions. D'une part, le domaine de la vie compris comme domaine de la morale s'appuie parfois sur la vérité : tout l'ouvrage Contre les moralistes (M XI) de Sextus Empiricus est précisément voué à réfuter la forme dogmatique du choix et du refus, c'est-à-dire la forme de vie qui fonde ses choix sur une idée de la nature des choses et en particulier la nature du bien et du mal. D'autre part, il peut arriver qu'une certaine forme de théorie ou de connaissance ne s'appuie pas sur la vérité mais renvoie à la pratique: Sextus range ainsi non seulement les affections mais aussi la sensation et la pensée, la connaissance des lois et des coutumes et l'apprentissage des arts sous la notion d'observation vitale ( $P H$ I 23-24). Il convient donc de bien garder la distinction entre les deux critères dans toute sa généralité afin de bien mesurer là où le critère devient dogmatique et là où il peut rester sceptique : partout où intervient un jugement de vérité ou de fausseté, il n'y a pas de scepticisme possible ; inversement, le jugement purement pratique, c'est-àdire sans attribution de vérité ou de fausseté, peut permettre de prendre des décisions dans tous les champs de la vie humaine, d'une manière compatible avec le scepticisme.

Cette première approche de la nature du critère sceptique, conçu comme critère d'action, permet de contester certaines lectures classiques de cette question. Premièrement, il est d'usage de considérer que la distinction entre critère d'action et critère de vérité est d'origine épicurienne et se 
retrouve donc dans des passages non sceptiques ${ }^{42}$. On renvoie généralement au passage de Diogène Laërce (DL X 34) dans lequel est exposé le critère épicurien que sont les affections $(\pi \dot{\alpha} \theta \eta)$, soit le plaisir et la douleur, «par

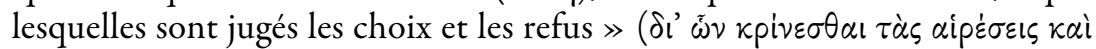
$\phi u \gamma \dot{\alpha} \varsigma)$. On notera cependant que ce critère est présenté un peu plus haut

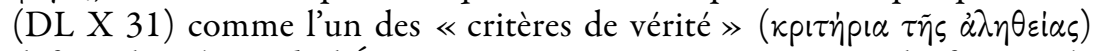
définis dans la Règle d'Épicure - expression qui témoigne du fait que le critère du choix et du refus n'est pas conçu comme critère d'action distinct du critère de vérité comme c'est le cas chez Sextus. Il en est de même pour le passage que l'on trouve chez Sextus Empiricus, vraisemblablement inspiré d'Épicure, sur les critères que Diotime attribue à Démocrite, dont le


tions $(\pi \dot{\alpha} \theta \eta)$, par différence avec les apparences, critère de l'appréhension des choses non manifestes, et avec la notion comme critère de la recherche ( $M$ VII 140) : le contexte du passage est celui de la présentation des thèses dogmatiques sur le critère, et il n'est absolument pas dit que Diotime fasse alors allusion à une distinction entre critère d'action et critère de vérité. Mais ne faut-il pas plutôt se référer aux textes d'Épicure lui-même, et non à des réécritures doxographiques ${ }^{43}$ ? Dans la Lettre à Ménécée, $\$ 129$, Épicure écrit que « c'est en partant <du plaisir> que nous faisons commencer tout


jugeant de tout bien avec l'affection comme règle ( $\dot{\omega} \varsigma \alpha \nu \dot{\nu} v \underline{\tau} \tau \tilde{\omega} \pi \dot{\alpha} \theta \varepsilon l \pi \tilde{\alpha} \nu$

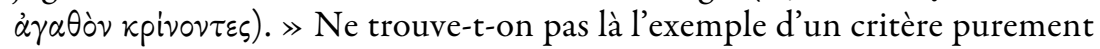
dédié à la conduite de la vie, sans considération de quelque vérité que ce soit ? Le problème est que, comme d'autres passages des Lettres le manifestent, l'affection ( $\pi \dot{\alpha} \theta_{o \varsigma}$ ) renvoie bien, chez Épicure, au vrai et constitue un critère au sens de ce qui permet d'atteindre les choses mêmes, ce qui d'ailleurs singularise cette doctrine philosophique par rapport à celles qui font de l'affection une pure voie de l'erreur ${ }^{44}$. Ainsi, chez Épicure, l'affection constitue une forme de noyau qui permet d'atteindre et de signaler la vérité, même si elle n'est pas un critère unique et suffisant, et qu'elle est complétée par la sensation et les préconceptions et projections de la pensée. Si c'est un critère du choix et du refus ou une règle du bien, ce n'est pas au

42. Voir Brunschwig 1995 [1988], p. 304 n. 2, qui renvoie à DL X 31-34 et À $M$ VII 140, etc. - repris, par exemple, par Corti 2009, p. 76 n. 1.

43. Je dois au relecteur anonyme de l'article cet ultime développement et les références aux passages d'Épicure.

44. Voir Lettre à Hérodote, 38 (où les «affections réelles 》 ( $\tau \dot{\alpha}$ i் $\pi \dot{\alpha} p x \circ v \tau \alpha \pi \dot{\alpha} \theta \eta)$ sont conçues comme des «critères » (кpı tnpia) au même titre que les préconceptions et projections de la pensée pour conférer aux choses leur caractère d'évidence), 63 (les sensations

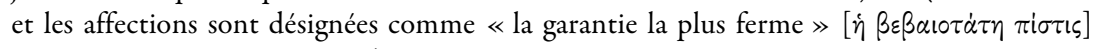
pour donner la nature de l'âme), 68 . 
sens où l'affection permettrait de juger dans le seul domaine de l'action mais c'est plutôt au sens où le choix et le refus constituent la base première qui permet d'attester du vrai et du faux ${ }^{45}$. L'affection, complétée parfois par le raisonnement ${ }^{46}$, est bien ce qui sert à agir, et l'on peut sans doute s'en tenir à cette fonction éthique qui permet de distinguer entre ce qui est bon et ce qui est mauvais pour nous : mais cette fonction éthique renvoie à un fondement vrai et à une épistémologie, à une théorie de la connaissance des choses, dont l'affection est l'une des pierres. Toutes ces précisions permettent de mesurer clairement la différence entre ce type de critère et le critère pratique tel que Sextus le conçoit, qui se distingue radicalement du critère de vérité. Pour le dire autrement, chez Épicure, le critère d'action est aussi un critère de vérité alors que, chez Sextus Empiricus, le critère d'action est fondamentalement autre qu'un critère de vérité.

Deuxièmement, contre l'idée que le sceptique se fonde tout uniment sur un critère d'action ou de vie, on rapporte le fait que, dans de nombreux passages sceptiques, la vie elle-même est conçue comme l'une des parties du désaccord entre les thèses qui conduit à la mise en suspens : la vie ne saurait donc être le fondement du jugement des sceptiques, et tout au plus les sceptiques se contredisent-ils sur ce point ${ }^{47}$. On peut par exemple renvoyer au passage de Sextus où l'existence des dieux est fondée sur la préconception commune de la vie ( $M$ IX 50 et 61) en opposition avec les nombreux arguments contre l'existence des dieux ( $M$ IX 51-59). Or, la réponse est à trouver dans le passage quelques paragraphes plus haut ( $M$ IX 49) qui explique que le sceptique, selon les lois et coutumes de ses pères, dit que les dieux existent (Eival), tout en ne se précipitant en rien pour autant qu'il s'agit de la recherche philosophique. Ce texte est un renvoi très clair à la distinction entre critère d'action et critère de vérité, même si l'usage du terme Eîval peut créer une légère confusion et un sentiment de contradiction : le sceptique se fonde dans ses jugements, par exemple à propos des dieux, sur le critère de vie, mais pas sur le critère de vérité car, de ce point de vue, le sceptique mettra en opposition les thèses inverses et restera

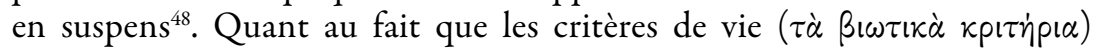

45. Cf. Sextus Empiricus, $M$ VII 203, qui est comme une forme de paraphrase de la doctrine épicurienne du critère telle qu'on la trouve exprimée dans les Lettres d'Épicure et dans la réécriture de Diogène Laërce.

46. Voir Lettre à Ménécée, 132 (qui pose la complémentarité de l'affection, critère des choix et des refus, et du raisonnement, qui recherche les raisons de ces choix et refus, pour bien vivre).

47. Voir Bailey 2002, chapitre 8, p. 175-213.

48. Cf. Brennan 1994, p. 159: «Universal agreement about the existence of gods is a criterion, in the sense of a reason for doing this rather than that. But it is not a criterion of truth in the sense of an infallible guide to a deeper reality, as certain Dogmatists would like to say. 》 
soient placés dans la catégorie des critères de vérité dans la typologie des critères (général, spécifique, très spécifique) proposée au début du livre II des Esquisses (PH II 15) et au début du Contre les logiciens ( $M$ VII 31-34), on pourra là aussi considérer que, en dépit d'une maladresse de formulation qui crée de la confusion, Sextus indique que les critères de vie (comme les sensations ou les différents instruments de mesure) peuvent être parfois utilisés comme critères de vérité : de ce point de vue, le sceptique leur opposera les arguments contre cette prétention au vrai, et restera en suspens. En revanche, ces mêmes critères de vie sont généralement utilisés sans attribution de vérité ou de fausseté, mais seulement pour faire des choix ponctuels dans telle ou telle situation : un tel usage sera parfaitement compatible avec le scepticisme ${ }^{49}$.

Afin de bien comprendre la portée de la conception du critère sceptique comme critère d'action, il paraît utile de proposer un double approfondissement de la compréhension de la notion. Premièrement, du côté de la différenciation par rapport au critère de vérité, il convient de revenir sur un autre aspect qui caractérise le point de vue dogmatique, complémentaire du renvoi à l'existence et à l'inexistence des choses: il s'agit de l'idée selon laquelle le critère de vérité, contrairement au critère d'action, constitue une garantie $(\pi i \sigma \tau \imath)$, comme l'exprime $P H$ I 21 . Cette notion est tout à fait technique dans le scepticisme: en effet, les textes de Sextus comportent comme une forme de leitmotiv l'idée que, pour un sceptique, les arguments ou les apparences sont « égaux du point de vue de la garantie ou de l'ab-

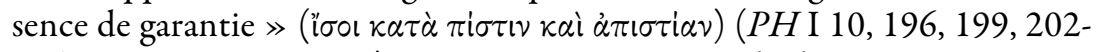
204, $M$ VIII 78, $M$ X 69). C'est même un moyen de distinguer sceptiques authentiques et pseudo-sceptiques. Ainsi, Platon, «lorsqu'il privilégie quelque chose selon sa garantie ou son absence de garantie, s'éloigne du caractère sceptique » $(P H \text { I 222 })^{50}$. Et, encore plus nettement, comme l'écrit Sextus un peu plus loin ( $P H$ I 227), «nous disons que les impressions sont égales du point de vue de la garantie ou de l'absence de garantie pour autant qu'il s'agit de l'argumentation, mais eux, <les académiciens $>$, disent que les unes sont convaincantes et les autres non convaincantes $»^{51}$. Outre le fait que ce texte met clairement en opposition

49. Voir Brennan 1994, p. 156-157. Contra Brunschwig 1995 (1988), p. 306-309, qui y voit le simple résultat de la juxtaposition de sources non concordantes dans les ouvrages de Sextus.

50. Cf. $P H$ I 223 : « dès lors que, d'une manière générale, il privilégie une impression sur une impression selon sa garantie ou son absence de garantie. »

51. Par différence, on notera que Sextus écrit à propos d'Arcésilas (PH I 232) qu'« il ne se trouve avoir rien déclaré à propos de l'existence ou l'inexistence de quoi que ce soit, ou n'avoir aucunement privilégié une chose sur une autre selon la garantie ou l'absence de garantie. » Arcésilas évite donc, comme un authentique sceptique, les deux dimensions 
l'attitude pyrrhonienne et l'attitude académicienne à l'égard de la garantie, l'expression « pour autant qu'il s'agit de l'argumentation » peut être comprise comme un renvoi au fait que le discours de la garantie est à inclure dans la partie réfutative du scepticisme, celle qui oppose argumentation contre argumentation, c'est-à-dire la partie qui concerne le critère de vérité, alors que, selon un autre point de vue, c'est-à-dire celui du critère d'action, une impression peut entraîner l'assentiment et conduire à faire tel ou tel choix. Il convient en effet de noter que, si le critère d'action ne saurait constituer une garantie $(\pi i \sigma \tau / \zeta)$, il est néanmoins ce à quoi l'on peut se ré-

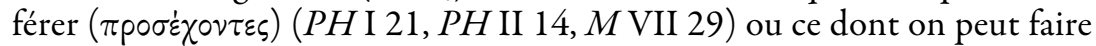


critère sceptique, le problème de la forme d'assentiment qu'un sceptique peut donner, ce que Cicéron nomme approbare $(A c \text {. II 104 })^{52}$ et Sextus ap-

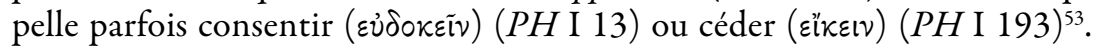
Deuxièmement, du côté de l'approfondissement de la compréhension de la notion de critère d'action, il faut souligner à quel point son application ne se limite pas au domaine de l'action stricto sensu. En effet, comme nous l'avons vu, il convient de relier la notion de critère d'action à celle

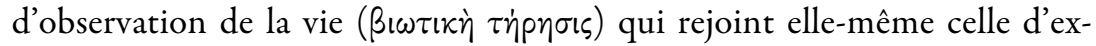
périence $(\dot{\varepsilon} \mu \pi \varepsilon i p i \alpha)$ et celle d'habitude $(\sigma \nu v \dot{\eta} \theta \varepsilon i \alpha)^{54}$. Or, ces notions permettent généralement de caractériser la manière dont un sceptique peut exercer son jugement, notamment dans le champ de la théorie, mais sans jamais tomber dans un discours de vérité et de faussetée ${ }^{55}$. Ainsi, alors qu'il

principales du critère de vérité, existence ou inexistence et garantie ou absence de garantie. À cet égard, on pourra s'étonner de ce que, en $M$ VII 158, Sextus rapporte que le critère d'action d'Arcésilas constitue une garantie ( $\pi i \sigma \tau i \varsigma)$ pour la vie heureuse : soit l'usage de ce terme est très maladroit, soit il constitue un indice supplémentaire de la dimension strictement dialectique de ce passage.

52. Voir Brittain 2006, p. XXVII et 61 n. 54.

53. Un texte de Galien, tiré de Sur la meilleure doctrine, $\$ 51$, est particulièrement intéressant à cet égard : « en rien il ne diffère sur ce qui est de la garantie des choses jugées

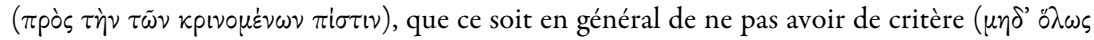

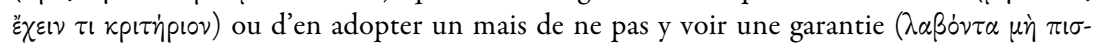

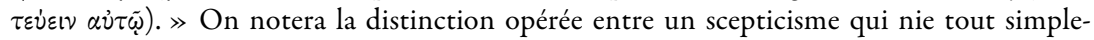
ment le critère et un scepticisme qui accepte un critère mais sans y voir une garantie. Un sceptique authentique nie le critère de vérité mais accepte un critère d'action sans en faire une garantie.

54. Sur cette dernière notion, qui apparaît tant chez Sextus ( $P H$ III 151) que chez Diogène (DL IX 105 et 108) pour nommer ce que le sceptique suit sans tomber dans le dogmatisme, voir Bett 2000 p. 89-90.

55. On notera quelques cas où la notion d'habitude $(\sigma \nu v \dot{\eta} \theta \varepsilon 1 \alpha)$ désigne l'une des branches de l'opposition dialectique par opposition à la philosophie ( $M$ VII 322, $M$ VIII 129); dans la plupart des textes du Contre les grammairiens ( $M$ I ), elle désigne le fondement 
met en évidence les impasses de la dialectique dogmatique pour résoudre des sophismes, Sextus rapporte la manière sceptique de s'en débarrasser.

Quant à nous, en partant sans opinion de l'observation de la vie ( $\dot{\alpha} \pi \grave{o} \tau \tilde{\eta} s$ $\beta \iota \omega \tau \iota \kappa \tilde{\eta} s \tau \eta p \dot{\gamma} \sigma \varepsilon \omega \varsigma)$, nous ne nous faisons pas entraîner par les discours trompeurs. (...) Ainsi, l'expérience de l'utile en chaque chose ( $\dot{\eta} \dot{\varepsilon} \nu \dot{\varepsilon} \alpha \dot{\alpha} \sigma \tau 015$

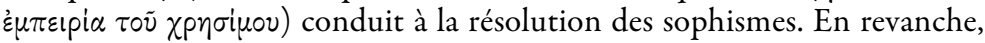
en ce qui concerne toutes les ambiguïtés qui ne se retrouvent pas dans l'une

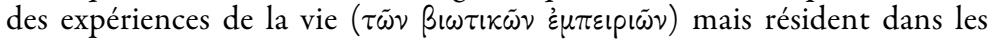
croyances dogmatiques et sont sans aucun doute inutiles pour vivre sans

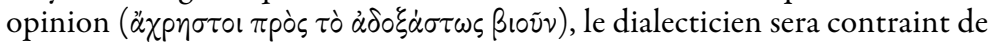
les laisser en suspens du fait des procédés sceptiques. (PH II 254-258.)

Ce texte permet de mettre en évidence le contraste entre l'efficacité du critère d'action, exprimé dans les notions d'observation de la vie et d'expérience, et la réduction à la suspension du critère de vérité que sont les croyances dogmatiques du dialecticien inutiles pour vivre, à propos d'un même objet, la dissolution des sophismes. Cet exemple montre de la manière la plus manifeste que les deux critères n'opèrent pas tant dans deux domaines distincts qu'ils n'abordent un même domaine de deux manières radicalement différentes.

On retrouve une même confrontation du critère d'action et du critère de vérité à propos de la manière dont il convier de procéder pour bien parler.

C'est pourquoi, si les grammairiens promettent de faire de la dite « analogie rationnelle » un art par lequel ils nous contraignent de converser conformément au bon langage, il convient de montrer l'inconsistance de cet art et il faut que ceux qui veulent correctement converser s'en remettent à l'observation toute simple et dépourvue d'art qui est conforme à la vie et à l'habitude commune du plus grand nombre $(\tau \tilde{\eta}$

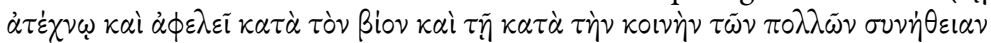
$\pi \alpha \rho \alpha \tau \eta \eta \dot{\sigma \varepsilon l)}$. (M I 179.)

Il peut sembler assez dogmatique de poser que le recours à l'analogie rationnelle est inconsistant, alors qu'un sceptique devrait sans doute plutôt dire qu'il ne sait si cette méthode est inconsistante ou non. Ou bien la formule est maladroite, ou bien elle témoigne à nouveau du fait que, dès lors qu'il n'y a pas de renvoi à une quelconque vérité ou fausseté mais seulement à ce qui peut être utile pour vivre à un moment donné, le sceptique peut

opposé à l'analogie rationnelle dans la science du langage; de même pour celle d'expérience ( $M$ VIII 191, M I 57-72), qui désigne alors un courant de pensée empiriste dogmatique distinct d'un courant rationaliste. Mais, comme pour la notion de vie (Bios), il convient de distinguer les passages où ils ont une valeur de vérité, sur laquelle un sceptique ne saurait se prononcer, et les passages où ils ont une valeur pratique. Voir Brennan 1994, p. 159-160. 
exprimer des jugements fermes ${ }^{56}$. On notera que, quelques lignes plus loin, Sextus écrira très explicitement que « l'habitude ( $\sigma \nu \nu \eta \dot{\theta \varepsilon l \alpha)}$ est le critère

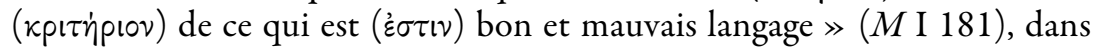
une formulation qui semble à nouveau glisser du critère d'action au critère

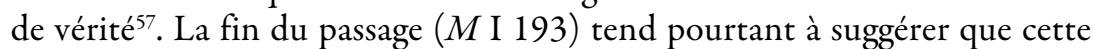
assertion est conforme à un principe général, celui de privilégier le critère d'action sur le critère de vérité : « sur presque toutes les commodités de la

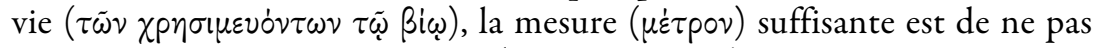

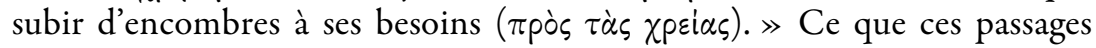
tendent à faire penser, c'est que, loin de ne pouvoir servir dans les domaines théoriques, le critère d'action tend même à damer le pion au critère de vérité, lequel est renvoyé tantôt à son caractère indécidable tantôt à son inutilité. Ainsi, le critère d'action, identifiable à l'observation de la vie, à l'expérience et à l'habitude, semblerait également s'identifier à l'utile. Le risque est que, à trop généraliser de la sorte, le sceptique ne retombe dans une forme de dogmatisme, qui voudrait que l'utile soit universellement ou par nature à choisir. Disons qu'il conviendra à chaque fois de préciser que l'utile ne se juge que dans des circonstances particulières et que ce qui peut paraître utile à l'un semblera inutile à l'autre. De cette manière, le critère d'action préservera peut-être sa qualité sceptique.

Au terme de cette analyse de la manière sceptique d'aborder le critère, on pourra soulever deux ordres de problème. Premièrement, est-ce que le scepticisme est une sortie de la philosophie et un retour à la vie ordinaire ? À cette question complexe, qui impliquerait de définir ce que l'on entend précisément par philosophie et par vie ordinaire, on pourra répondre sous forme d'esquisse en deux temps. D'une certaine manière, le critère d'action du sceptique est bien conçu comme un critère fondamentalement non philosophique, au sens général où il ne renvoie pas à la recherche du vrai. C'est ce qui fait que Sextus, répondant à l'objection dogmatique de l'incapacité pour un sceptique de faire des choix, expliquait que le sceptique est incapable d'agir s'il en reste au point de vue philosophique, car c'est le lieu du conflit des opinions et de la mise en suspens, alors qu'il fera des choix quand il se placera du point de vue non philosophique, c'est-à-dire du point de vue de l'observation de la vie (M XI 165). Mais Sextus peut parfois énoncer des propos d'une hostilité virulente à la philosophie.

Quant à moi, je pense qu'il suffit de vivre ( $\beta$ เoũ) de manière empirique

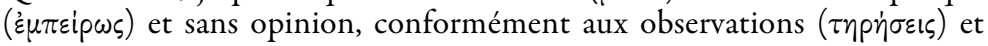

56. Sur tout le passage $M$ I 179-193 et son caractère sceptique ou dogmatique, voir Corti 2009, p. 206-219.

57. Pour Corti 2009, p. 217-218, il y aurait en $M$ I 181-189 de nombreux glissements du critère d'action au critère de vérité, qui divergeraient des formulations de $P H$ I 21-24. 
préconceptions communes, en restant en suspens sur ce qui est dit du fait de la superfluité dogmatique et surtout en dehors de toute utilité pour la

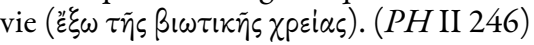

Des passages de ce genre (cf. $M$ VIII 158) alternent avec d'autres qui se contentent tout au plus de mettre en opposition vie et philosophie, sans trancher sur la question de savoir quel point de vue est à choisir ( $M \mathrm{X} 49$ ). Le point important à rappeler, à cet égard, est qu'un sceptique ne prendra fait et cause pour une position que du point de vue d'un critère pratique et non du point de vue d'un critère de vérité. C'est ce qui amène à répondre aussi que la conception sceptique du critère implique une attitude qui est tout sauf ordinaire et dépourvue d'un sens philosophique : en effet, une vie accompagnée de la pensée permanente que « je ne sais pas si ce qui m’apparaît est réellement le cas » et de «c'est ce qui m'apparaît à moi dans ces circonstances relatives données $\gg$ est une vie tout sauf ordinaire ${ }^{58}$. Mais on pourra poser un deuxième ordre de problèmes. Nous avons vu que le sceptique, s'il conserve une forme de critère, appelle à se débarrasser de toute attribution de la vérité. Mais, qu'est-ce qu'une vie d'homme sans vérité ? L'argument peut prendre deux formes. D'une part, il sera rétorqué au sceptique que la vérité est la base indispensable de toute vie. Les interprètes contemporains, selon leurs obédiences philosophiques profondes, sont ici divisés ${ }^{59}$. On pourra aussi renvoyer à un passage de Galien (De compositione medicamentorum secundum locos, Kühn XIII, p. 116, 1. 5) qui semble conditionner la possession d'un critère d'action à la reconnaissance de la possibilité du vrai : «à tous les êtres sensés et qui chérissent réellement la vérité, je souhaite de veiller sur les critères dont nous avons été dotés par nature pour les actions de la vie, à savoir l'expérience et la raison. » On notera que Sextus, en $P H$ I 23-24, articulera justement le fait de rester en suspens sur la vérité des choses et le fait de reconnaître l'indication de la nature que sont la sensation et la pensée comme des critères d'action. D'autre part, qu'est-ce qu'une vie d'homme sans vérité, au sens où la vérité serait le domaine d'exercice de l'homme accompli ? Ce point est

58. Cf. Mates 1996 p. 74 : « [a Pyrrhonist] would insist that adopting Pyrrhonism should make no difference to action, and that in particular it needs make no difference to patterns of speech. If it makes any difference at all, the difference will be an "internal" one, relating specifically to a protophilosophical belief in a so-called "external world" of things and facts. »

59. Voir Burnyeat 1979 ou Barnes 1982 versus Mates 1996, p. 15: «when the Pyrrhonist says that he uses the appearances as criteria for action, he means only that at every time he goes by what appears to him to be the case and does not concern bimself with any question of what really is the case - ou Vogt 2010, p. 170: «the sceptic can call into question the plausibility of the Stoic assumption that, in everything we do, we judge things to be so-and-so. Perhaps the sceptic, in merely "adhering" to impressions, is actually rather like everyone else, and it turns out to be a dogmatic fantasy that action ordinarily involves assent. » 
également suggéré par Galien, dans un passage de Des sectes pour les débutants (chapitre VII, $\$ 16$ ), où il distingue entre deux types de critère, la raison et les apparences, en précisant que le premier critère s'adresse aux non-débutants, tandis que le second «est commun à tous les hommes ». Ainsi, comme il l'énonce plus loin, « en posant l'apparent comme règle de

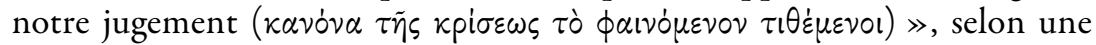
formulation qui résonne avec celles de Diogène, de Sextus mais aussi de Galien lui-même sur le critère sceptique, nous en restons au niveau de l'homme ordinaire. C'est le niveau de la raison, celui qui ne se contente pas de la succession des apparences, qui est celui de l'homme supérieur. Cette thématique, que l'on retrouve dans certains passages des Entretiens d'Épictète (livre II, chapitre 11), n'est pas non plus étrangère à Sextus Empiricus, lequel rapporte, au début des Esquisses (PH I 12), que « les hommes d'une nature supérieure 》 ( $\mu \varepsilon \gamma \alpha \lambda \circ \phi v \varepsilon i \bar{\zeta} \tau \tilde{\omega} \nu \dot{\alpha} \nu \theta \rho \omega \dot{\omega} \pi \omega \nu)$, troublés par l'irrégularité des choses, en vinrent à chercher le vrai et le faux. Le point de savoir comment un sceptique répondrait à l'accusation d'empêcher le plein déploiement de l'humanité rejoint donc le statut de la recherche de la vérité dans le scepticisme ${ }^{60}$. Ce que l'on pourra se contenter de dire, à ce stade, est que, comme nous l'avons souligné, le sceptique ne détruit pas le critère de vérité mais il se contente de s'interroger sur la possibilité de l'établir et de renvoyer toujours de nouveaux arguments qui viennent éloigner cette possibilité. C'est une autre manière de dire que la recherche de la vérité est sans cesse recommencée, mais que cette quête indéfinie n'est cependant pas un obstacle pour vivre. 


\section{BIBLIOGRAPHIE}

ANNAS, J. 1986: «Doing without objective values : ancient and modern strategies », dans M. Schofield \& G. Striker (éd.), The Norms of Nature: Studies in Hellenistic Ethics, Cambridge-Paris, 1986, p. 3-29.

- 1990 : « Stoic Epistemology », dans S. Everson (éd.), 1990, p. 184-203.

BAILEY, A. 2002 : Sextus Empiricus and Pyrrhonean Scepticism, Oxford, 2002.

BARNeS, J. 1982 : «The Beliefs of a Pyrrhonist », Proceedings of the Cambridge Philological Society, New series, 28 (1982), p. 1-29.

- 1990 : « Pyrrhonism, Belief and Causation. Observations on the Scepticism of Sextus Empiricus », Aufstieg und Niedergang der Römischen Welt, II, 36.4, Berlin, 1990, p. 2608-2695.

- 2007: « Sextan Scepticism », dans D. Scott (éd.), Maieusis : Essays on ancient philosophy in honour of Myles Burnyeat, Oxford, 2007, p. 322-334.

BETT, R. 1989 : « Carneades' Pithanon : a reappraisal of its role and status », Oxford Studies in Ancient Philosophy, 7 (1989), p. 59-94.

- 2000 : Pyrrho - His antecedents and his legacy, Oxford, 2000.

- 2005 (trad.) : Sextus Empiricus. Against the Logicians, Cambridge, 2005.

- 2010 (éd.) : The Cambridge Companion to Ancient Scepticism, Cambridge, 2010 (Cambridge Companions to Philosophy).

Brennan, T. 1994 : «Criterion and Appearance in Sextus Empiricus : the scope of sceptical doubt, the status of sceptical belief », Bulletin of the Institute of Classical Studies, 39 (1994), p. 151-169.

- 1998 : « Pyrrho on the Criterion », Ancient philosophy, 18/2 (1998), p. 417-434.

BritTAIN, C. 2006 (trad.) : Cicéron. On Academic Scepticism, Indianapolis, 2006.

BROCHARD, V. 1887 : Les Sceptiques grecs, Paris, 1887 [réimpr. Paris, Livre de poche, 2002].

BRUNSCHWIG, J. 1995 : «Le problème de l'héritage conceptuel dans le scepticisme : Sextus Empiricus et la notion de kriterion» (1988), dans Études sur les philosophies bellénistiques, Paris, 1995, p. 289-319.

BURNYEAT, M. 1979 : « Can the sceptic live his scepticism ? », dans M. Schofield, M. Burnyeat et J. Barnes (éd.), Doubt and Dogmatism, Oxford, 1979, p. 20-53.

CORTI, L. 2009 : Scepticisme et langage, Paris, 2009.

EVErson, S. 1990 : Companions to Ancient Thought 1: Epistemology, Cambridge, 1990 (Companions to Ancient Thought).

Hankinson, R. J. 1995 : The Sceptics, Londres-New York, 1995.

- 2010 : « Aenesidemus and the rebirth of Pyrrhonism », dans R. Bett (éd.), 2010, p. 105119.

Huby P. et G. NEAL 1989 (éd.) : The Criterion of Truth : essays written in honour of George Kerferd together with a text and translation (with annotations) of Ptolemy's "On the kriterion and hegemonikon", Liverpool, 1989.

IOppolo, A.-M. 2009: La testimonianza di Sesto Empirico sull Academia scettica, Naples, 2009.

JANÁČEK, K. 1947 : Prolegomena to Sextus Empiricus, Olomouc, 1947.

- 1972: Sextus Empiricus' sceptical methods, Prague, 1972.

LÉVY, C. 2008 : Les scepticismes, Paris, 2008.

LONG, A. 1978 : « Sextus Empiricus on the criterion of truth », Bulletin of the Institute of Classical Studies 25/1 (1978), p. 35-49.

-1989 : « Ptolemy on the criterion : an epistemology for the practising scientist », dans P. Huby \& G. Neal (éd.), 1989, p. 151-178.

MATES, B. 1996 (trad.) : Sextus Empiricus. The Skeptic Way: Sextus Empiricus's Outlines of Pyrrhonism, New York, 1996. 
Pellegrin, P. 1998 (trad.) : Galien. Traités philosophiques et logiques, Paris, 1998.

- 2010 : «Sextus Empiricus », dans R. Bett (éd.), 2010, p. 120-141.

PERIN, C. 2010 : The demands of reason - An essay on pyrrhonian scepticism, Oxford, 2010.

POLITO, R. 2007 : « Was skepticism a philosophy ? Reception, self-definition, internal conflicts », Classical Philology, 102/4 (2007), p. 333-362.

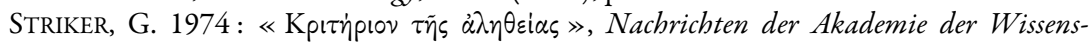
chaften in Göttingen, 2 (1974), p. 47-110.

- 1990: «The problem of the criterion », dans S. Everson (éd.), 1990, p. 143-160.

- 2010 : «Academics versus Pyrrhonists, reconsidered », dans R. Bett (éd.), 2010, p. 195207.

Svavarsson, S. H. 2010 : «Pyrrho and early Pyrrhonism», dans R. Bett (éd.), 2010, p. 36-57.

ThorsRud, H. 2010 : « Arcesilaus and Carneades », dans R. Bett (éd.), 2010, p. 58-80.

VOGT, K. M. 2010 : «Scepticism and action », dans R. Bett (éd.), 2010, p. 165-180. 